\title{
Efficient Morphometric Techniques in Alzheimer's Disease Detection: Survey and Tools
}

\author{
Vinutha N., P. Deepa Shenoy and K.R. Venugopal \\ Department of Computer Science and Engineering, University Visvesvaraya College of Engineering, India
}

Corresponding Author:

Vinutha $\mathrm{N}$.

Department of Computer

Science and Engineering,

University Visvesvaraya

College of Engineering, India

Email: vinutha1v@gmail.com

\begin{abstract}
The development of advance techniques in the multiple fields such as image processing, data mining and machine learning are required for the early detection of Alzheimer's Disease (AD) and to prevent the progression of the disease to the later stages. The longitudinal and cross sectional images of elderly subjects were obtained from the standard datasets like ADNI, OASIS, MIRIAD and ICBM. The subject image obtained from the dataset, can be geometrically aligned to the template image through the process of registration. The registration techniques like Mutual Information Registration, Fluid registration, Rigid registration, Spatial Transformation algorithm for registration, Elastic Registration are selected based on type of transformation and similarity measures to suit the required application. The registered images are then subjected to the process of segmentation in order to segment relevant tissues or desired region of interest that are significant in AD detection. The different types of segmentation techniques such as Tissue Segmentation, Atlas based Segmentation, Hippocampus Segmentation and other segmentation techniques have been discussed. The segmented images are then subjected to morphometry techniques to identify the morphological changes developed in an abnormal image. The different types of morphometry techniques used are Voxel Based Morphometry (VBM), Deformation Based Morphometry (DBM), Shape Based Morphometry (SBM) and Feature Based Morphometry (FBM). But in recent years, the main focus of researchers is towards the FBM and SBM to overcome the disadvantage of group analysis that existed in VBM and DBM. Further the data is classified into healthy normal and $\mathrm{AD}$ by supervised, unsupervised or probabilistic methods.
\end{abstract}

\section{Keywords: Alzheimer's Disease, Classification, Morphometry, Registration, Segmentation}

\section{Introduction}

The broad term neurodegenerative represents a group of disorder, which are characterized by the degeneration of structure and functions of neurons. Examples of neurodegenerative diseases that are progressive and incurable include Alzheimer's Disease, Parkinson's Disease, Amyotrophic lateral sclerosis and Huntington's Disease. Alzheimer's Disease (AD) is the most common neurodegenerative disease that occurs among the older adults. It is caused by the deposition of Amyloid Plaques and Neurofibrillary Tangles in the different regions of the brain. These pathological changes alter the structures and functionalities of the brain, leading to cognitive and behavioral impairment. The changed brain structure can be acquired through different image modalities such as Magnetic Resonance Imaging (MRI), Cerebrospinal Fluid (CSF), Single Photon Emission Computed Tomography (SPECT) and Fluorodeoxyglucose Positron Emission Tomography (FDG-PET). The image processing techniques are required to optimize an image, which is essential to improve the pictorial representation of an image and the machine perception and also to aid in human visual interpretation. The optimized image is further subjected to different data mining techniques in order to get into deeper insights of data and to mine the relevant information. The extracted relevant information 
determine the changed pattern in the abnormal brain. The other types of data such as Demographic Information, Clinical Scores, Genotypic Information and Fluid Biomarkers are integrated with the images to improve the prediction of $\mathrm{AD}$.

\section{Stages of Alzheimer's Disease}

The progression of $\mathrm{AD}$ comprises of three different stages:

- Preclinical Alzheimer's Disease: It is a preliminary stage of $\mathrm{AD}$, where the symptoms are not developed in the affected patient at the early stage. Thus the early state of $\mathrm{AD}$ is identified through the Imaging and the Fluid Biomarkers

- Mild Cognitive Impairment: Mild Cognitive Impairment (MCI) is an intermediate stage between normal and AD. The MCI patients have mild memory and cognitive problems, however they are capable of executing daily activities. The two different types of MCI are Amnestic Mild Cognitive Impairment (aMCI) and non-amnestic Mild Cognitive Impairment (naMCI)

- Alzheimer's Disease: This is the most critical stage, where the person is incapable of performing day to day activities because of loss of memory and reduction in thinking ability and changes in behaviour

\section{Symptoms of Alzheimer's Disease}

- Inability to remember recent events

- Decreased ability to recognise others

- Difficult to make decision on personal activities

- Difficult to plan and execute daily activities

- Depression

- Changes in sleep pattern

- Extreme periods of anger

- Challenges in solving simple problem

- Repetition of words

Alzheimer's Disease can be delayed by following an healthy diet, doing regular physical and mental exercises, proper sleep, stress management and maintaining social relationships.

\section{Motivation}

$\mathrm{AD}$ is the most common neurodegenerative disease that occurs among the elderly individuals. It has been predicted that millions of people can get affected in the future years. This motivates the researchers to focus their attention towards the advancement in the development of techniques required for accurate detection and diagnosis of $\mathrm{AD}$.

\section{Organisation}

The paper is organized as follows: Model Architecture is presented in Section II. Standard Dataset that is required to obtain Cross Sectional and Longitudinal images is explained in Section III. Brain Regions Affected due to Alzheimer's Disease are described in section IV. Segmentation Techniques are presented in Section V. Registration Techniques are described in Section VI. Morphometry Techniques are described in Section VII. Classification Techniques are explained in Section VIII. Tools available for Alzheimer's Disease detection are described in Section IX.

\section{Model Architecture}

The Model Architecture of the survey paper is discussed and shown in Fig. 1:

\section{Dataset}

The complementary information required for $\mathrm{AD}$ detection is obtained from structural and functional images, that aid to determine the abnormality of the affected brain. The other type of information such as clinical, genetic, demographic are also provided along with the image information by several standard datasets like ADNI, ICBM, MIRIAD and OASIS.

\section{Segmentation}

Segmentation is a key step to obtain the desired tissue or a particular region of interest from the collected images. Different types of segmentation techniques like Tissue Segmentation, Atlas-Based Segmentation, Hippocampus Segmentation and other automated segmentation techniques are discussed.

\section{Registration}

The standard dataset contains multiple images for the same subject that is acquired at different time points and with different modalities. All the images are aligned to a common reference space through the process of registration. The alignment provides intra-subject and intersubject matching that is required to study the disease progression and to identify abnormal subjects. The different types of registration techniques such as Mutual Information Registration, Fluid Registration, Rigid Registration, Spatial Transformation Algorithm for Registration, Elastic Registration are discussed.

\section{Morphometry}

Morphometry identifies the anatomical differences between normal and affected population. Different types of morphometry techniques such as Voxel-based Morphometry, Deformation-based Morphometry, Shape-based Morphometry and Feature based Morphometry are discussed. 


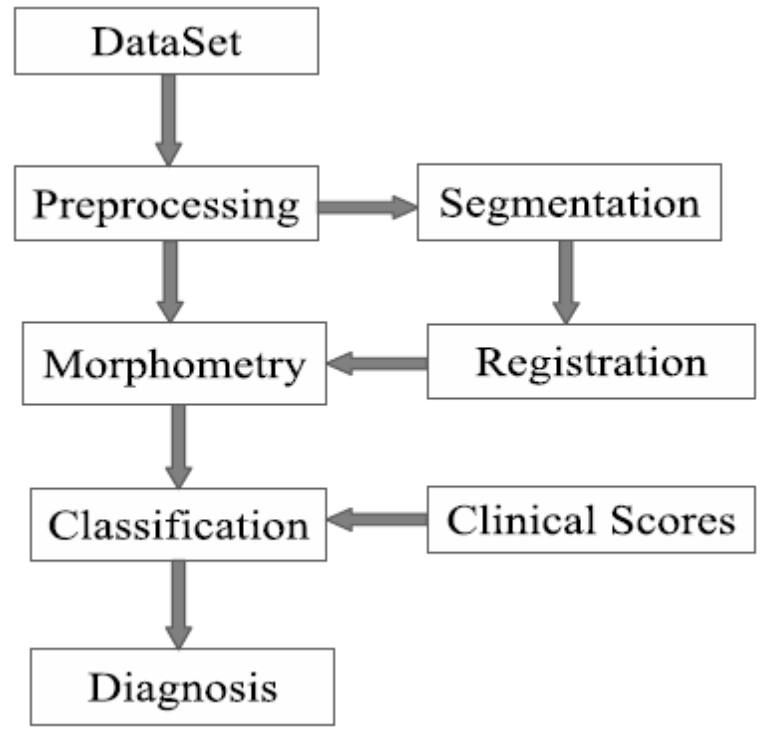

Fig. 1. Automatic Detection of Alzheimer's Disease

\section{Classification}

The classification is the last step required to classify the subjects into different classes based on normality and abnormality. The different types of classification techniques like supervised, unsupervised and probabilistic methods are discussed.

Tools

Numerous tools such as 3D SLICER, ABEAT, DRAMMS, FSL, FREE SURFER, HAMMER, MIPAV, SPM, VBM, AIR and IMAGEJ are developed to handle the techniques of preprocessing, registration, segmentation, morphometry and classification.

\section{Datasets}

The standard datasets like ADNI, ICBM, MIRIAD, OASIS provide the clinical, demographic and imaging information of older adults.

\section{ICBM}

International Consortium Brain Mapping (ICBM) (Mazziotta et al., 2001), consists of demographic, clinical, behavioural and imaging information of the older adults. The demographic information includes age, gender, education, family history and background. Clinical and behavioral information includes the psychiatric screening, neuropsychological tasks and neuropsychological test. T1, T2 weighted and proton density are the different multispectral MRI images gathered for the same subject. The main aim of this database is to develop disease specific probabilistic atlas and reference system of brain for Alzheimer's, Multiple Sclerosis, Schizophrenia and Stroke Disorder.

\section{OASIS}

Open Access Series of Imaging Studies (OASIS) (Marcus et al., 2007), is a dataset that provides 416 cross-sectional subjects between the age of 18-96 Years. It comprises of clinically diagnostic information of 100 older adults who are more than 60 years of age, affected with mild-moderate $\mathrm{AD}$ and it also includes nondemented subjects. The procedure of automatic brain volume computation and total intra cranial volume estimation are described, to indicate the usage of data in identification of the difference existing between Normal and $\mathrm{AD}$. There is a free access to the dataset for improving the early detection of AD.

\section{MIRIAD}

The Minimal Interval Resonance Imaging in Alzheimer's Disease (MIRIAD) (Malone et al., 2003), consists of T1 weighted longitudinal MRI scans with different sequence of intervals for the subject with mildmoderate $\mathrm{AD}$ and the normal controls. Furthermore, it is extended with the information of Mini-Mental State Examination (MMSE), age and gender. The data are made publically available to the researchers to measure the longitudinal volume change in the series of MRI scans.

\section{$A D N I$}

Alzheimer's Disease Neuro imaging Initiative (ADNI) (Jack et al., 2008), is a longitudinal multi site observational study of healthy elders, MCI and AD. It consists of information of different imaging modalities such as MRI, PET, CSF biomarker. In addition to these modalities, clinical and demographic information are added.

\section{BRAIN WEB}

A set of realistic simulated brain MR image volumes are obtained by varying specific imaging parameters and artifacts in MRI simulator. The parameter settings available for precomputed simulated database are fixed with three modalities, six levels of noise, three levels of intensity non-uniformity and five slice thickness. But the simulation with arbitrary parameters are executed by using brain web custom MRI simulations interface (Cocosco et al., 1997).

\section{Brain Regions Affected Due To Alzheimer's Disease}

The neurodegenerative disease leads to pathological changes in the different regions of brain, even before the development of symptoms. So it helps in detecting the present condition of disease and to predict risks in later stages. Different regions of brain, affected due to AD are listed in Table 1 and the description and functionalities of each region are explained below: 
Table 1. Regions affected due to Alzheimer's disease

\begin{tabular}{lll}
\hline Region & Location & Effects due to Alzheimer's disease \\
\hline Hippocampus (De Leon et al., 1993) & Medial temporal Lobe & Shrinkage \\
Amygdala (Brambilla et al., 2008) & Frontal portion of temporal lobe & Shrinkage \\
Entorhinal cortex (Bobinski et al., 1999) & Ventromedial surface of temporal lobe & Shrinkage \\
Ventricle (Nestor et al., 2008 ) & Core of the brain & Enlargement \\
Precuneus, posterior cingulate gyrus & Medial parietal lobe & Reduced metabolism \\
\hline
\end{tabular}

\section{Hippocampus}

Hippocampus (De Leon et al., 1993) is identified in the medial temporal lobe which are surrounded by the cortical regions. It is crucial for memory consolidation in the process of converting shortterm memory into long-lasting memory in the neocortex. The shrinkage of this region is induced more in age related problems due to its role in central memory. It is also affected by various disorders like Schizophrenia, Epilepsy, Stress and Mood disorder.

\section{Amygdala}

Amygdala (Brambilla et al., 2008) is closely associated with the hippocampus in the frontal portion of the temporal lobe. It connects to several cortical regions, receiving input from the frontal and temporal lobes and sending output information to limbic areas such as thalamus, entorhinal cortex, hippocampus and neocortex. It is a region which controls fear, emotion and anxiety.

\section{Entorhinal Cortex}

Entorhinal Cortex (Bobinski et al., 1999) is located below the hippocampus, in the ventromedial surface of the temporal lobe. It is actively involved in memory formation, retrieval and extinction as part of circuit involved. This is the first region affected in Mild Cognitive Impairment before developing to AD.

\section{Ventricles}

The location of ventricles (Nestor et al., 2008) are determined in the core of the brain which is composed of Cerebrospinal Fluid. Enlargement of lateral ventricle is due to various disorders such as AD, Schizhophrenia, Dementia and Bipolar disorder.

\section{Putamen}

Putamen (De Jong et al., 2008) is a paired structure located at the base of the forebrain. Putamen and caudate nucleus together form corpus striatum. The important functionalities of putamen are probabilistic learning, memory task and regulating movements.

\section{Thalamus}

Sensory and motor mechanisms are served by thalamus (De Jong et al., 2008). Atrophy of thalamus has also been associated with cognitive decline in neurodegenerative disorders such as Multiple Sclerosis, Huntington's Disease and Lewy Body Dementia.
The following papers discuss about the regions that are affected during different stages of $\mathrm{AD}$ :

MRI measures of the banks of superior temporal sulcus, the entorhinal cortex and the caudal portion of the anterior cingulate are useful in discriminating three different stages of AD (Killiany et al., 2000). The different stages are: Individuals with mild memory impairment which converts to $\mathrm{AD}$ in 3 years duration, individuals with mild memory problems without the advancement to $\mathrm{AD}$ in 3 years and normal controls.

Using multiple MRI scans, the gray matter atrophy pattern observed in amnestic Mild Cognitive Impairment (aMCI) before 3 years of AD diagnosis is on the medial temporal lobes such as amygdala, anterior hippocampus, entorhinal cortex and fusiform gyrus. Before 1 year of $\mathrm{AD}$ diagnosis, atrophy is induced in the middle temporal gyrus and extended to posterior regions of the temporal lobe, entire hippocampus region and parietal lobe. During diagnosis, the atrophy of the medial temporal lobe, the temporoparietal association cortices and the frontal lobe are affected much more (Whitwell et al., 2007).

Longitudinal studies (Teipel et al., 2003) indicate the decline in memory for an affected person by using time series data of the subject. The significant biomarkers observed during the diseased state are larger ventricular volume, decreased gray and white matter volumes in temporal and parietal lobes of brain. But the parietal lobe is an effective biomarker which shows significant changes when age factor is considered.

\section{Automatic Segmentation}

Segmentation is an important step required to obtain specific region of interest. Noise, limited resolution, partial volume effect are certain challenges to be focused during segmentation of medical images (Vibha et al., 2007). Due to $A D$, the progressive changes are observed with different regions or tissues of brain. This distinguishable regions or tissues aids the doctor to decide whether the person is normal or abnormal and it also improves the efficiency of the computer aided diagnosis. Different segmentation techniques, their advantages and limitation are discussed in Table 2.

The steps involved in edge detection technique are filtering, enhancement and detection. The performance of soft computing (Venugopal et al., 2009; Senthilkumaran and Rajesh, 2009) approaches like genetic algorithm (Shenoy et al., 2005) and neural 
network show better edges than robert, sobel and prewitt edge detectors.

Mumford-shah functional (Chan and Vese, 2001) is the new model of active contour with the techniques of curve evolution, for detecting the objects without the edges. It performs well even with noisy images by starting only with one initial curve. The location of initial curve need not be on object to be detected.

An automatic segmentation of Hippocampus (HC) and Amygdalae (AG) is obtained by the fusion of Active Appearance Modeling (AAM) and level set shape modeling (Hu et al., 2011). The fusion technique is useful to segment the MRI images, having weak intensity contrast with their background. The appearance model applied on multicontrast images such as T2, T1 and proton density weighted images improve the ROI segmentation.

An approach of image segmentation by histogram thresholding (Tobias and Seara, 2002) groups similar gray levels in an image according to predefined criteria and it is assessed by fuzzy Measure (Srinivasa et al., 2005). Since the method is not based on the minimization of a criterion function, it improves the result on bimodal and multimodal histogram. But it is limited to images that satisfy the assumption that the objects and background must occupy non-overlapping regions of the histogram.

A coupled surface propagation (Zeng et al., 1999) using level set method is proposed to obtain the geometric measurements of cortex (cortical surface curvature, cortical thickness map, cortical gray matter volume and cortical surface area). The advantage of the proposed technique: efficient computation, easy initialization, volumetric analysis and it has the potential to capture deep sulcal folds.

The thresholding is the popular method of image segmentation to discriminate the object of varying intensity. A multilevel threshold allows the determination of appropriate number of thresholds as well as the adequate threshold values by using the combination of Genetic Algorithm (GA) and Wavelet
Transform (WT) (Hammouche et al., 2008). The WT reduces the length of the histogram and GA determines the threshold values for the obtained histogram. The accuracy of image segmentation by thresholding is evaluated through the uniformity measure and the cost function.

\section{Tissue Segmentation}

The brain is made up of three different tissue types namely Gray Matter (GM), White Matter (WM) and Cerebrospinal Fluid (CSF) as shown in Fig. 2. In AD patients, atrophy of GM is more compared to WM and CSF. So it is necessary to perform tissue segmentation.

Fully automated intensity-based algorithms exhibit high sensitivity to noise artifacts, such as intra tissue noise, inter tissue intensity contrast reduction and partialvolume effects. Tissue segmentation by Constrained Gaussian Mixture Model (CGMM) (Greenspan et al., 2006) outperforms EM-based segmentation algorithm of Van-Leemput (KVL) under varying noise. CGMM combines local spatial information with global intensity modelling, which is an alternative to Markov Random Field. Prior information required for tissue segmentation is not considered, but the intensity order of tissues in MR image is considered. Processing time required for the segmentation is correlated to the number of Gaussians and the number of voxels in an image.

Brain is composed of various regions, where each region is formed by different types of tissue. The EM algorithm (Van Leemput et al., 2003) estimates missing parameters such as sub voxel intensity and subvoxel labels by utilising only the image intensity. In addition to this, down sampling is introduced to aggregate the subvoxel intensities and to develop the observed intensity. The estimated model parameters assign each voxel to a particular tissue, thereby resulting in the tissue classification. Using this algorithm, model parameter estimation is well determined in the low resolution MR images. Improved spatial model needs to be used as prior information for robust PV segmentation.

Table 2. Segmentation techniques for Alzheimer's disease detection

\begin{tabular}{|c|c|c|c|}
\hline Authors & Algorithm & Advantage & Disadvantage \\
\hline Coupé et al. (2011) & $\begin{array}{l}\text { Patch based } \\
\text { segmentation }\end{array}$ & $\begin{array}{l}\text { Non local means estimator } \\
\text { improve the segmentation }\end{array}$ & $\begin{array}{l}\text { The combination of non local } \\
\text { means and non linear registration } \\
\text { is to be focused in the future }\end{array}$ \\
\hline Zarpalas et al. (2014) & $\begin{array}{l}\text { Gradient distribution } \\
\text { boundary }\end{array}$ & $\begin{array}{l}\text { Increased dice } \\
\text { coefficient metric }\end{array}$ & $\begin{array}{l}\text { Future focus to introduce bias } \\
\text { correction concept and to identify } \\
\text { white matter borders }\end{array}$ \\
\hline Ribbens et al. (2014) & $\begin{array}{l}\text { Expectation and } \\
\text { maximization algorithm }\end{array}$ & Improved average dice overlap & $\begin{array}{l}\text { To determine the optimal no of } \\
\text { clusters automatically }\end{array}$ \\
\hline Sanroma et al. (2014) & $\begin{array}{l}\text { Histogram Oriented gradient, } \\
\text { SVM and majority voting }\end{array}$ & Improved Average dice ratio & To evaluate on other dataset \\
\hline Yazdani et al. (2016) & Hybrid Segmentation & $\begin{array}{l}\text { Superior performance on } \\
\text { white matter tissue }\end{array}$ & $\begin{array}{l}\text { To improve segmentation } \\
\text { of Gray Matter tissue }\end{array}$ \\
\hline
\end{tabular}




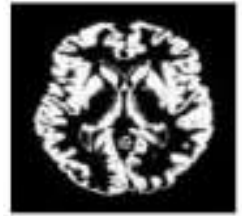

Gras Matter

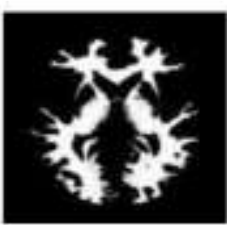

White Mlatter

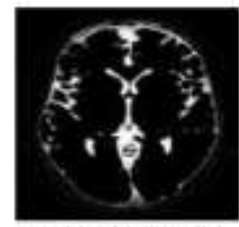

Cerebro Spinal Fluia
Fig. 2. Tissue segmentation for Alzheimer's disease detection

Ribbens et al. (2014) proposed unsupervised segmentation and construction of probabilistic atlases on the heterogeneous set of brain images obtained from the ADNI and BRAIN WEB dataset. For the given image, probabilistic atlas is constructed immediately after the segmentation and simultaneously clustered into a subgroup of images based on the selected atlas. This results in improved segmentation accuracy. The morphological patterns obtained for each subgroup are compared with the clinical data for specific diseases to evaluate the proposed framework. Registration of images to the atlases and developing automated methods for clustering the subgroups are to be focused in the future.

Segmentation results are improved by incorporating the spatial information into membership function. The likelihood of a pixel belonging to a particular cluster is represented through the spatial function and defined by the squared window. Then the membership function are computed to obtain cluster distribution statistics (Chuang et al., 2006).

A hybrid approach is developed to combine the advantages of region based and threshold-based methods on MRI brain images (Yazdani et al., 2016). The segmentation of MRI into three different tissues is achieved by combining both spatial and intensity information. The performance is evaluated by the calculation of AOM index for each tissue and the results indicate its superior performance in white matter tissue segmentation. Improvements should be focused on the Gray Matter tissue segmentation.

An adaptive mean shift methodology (Mayer and Greenspan, 2009) classifies brain voxels into three tissue types. The clustering technique is based on the joint intensity-spatial distribution of voxels, to extract highdensity points from the feature space using multi modal MRI data (T1, T2, Pd). Based on intensity mode, each voxel is clustered to one of the tissue types. This technique does not consider the spatial information prior from atlas but performs well with non-convex clusters to produce convergence mode for intensity based classification. This shows better results on noisy and biased data and can be further extended to detect abnormal tissue.

\section{Atlas Based Segmentation}

The prior information of anatomical surfaces of the brain are considered as an atlas. The atlas-based segmentation is most suitable for images, with no well- defined relation between regions and pixels intensities. It determines the morphological differences existing between the patient and normal brain as shown in Fig. 3. (Lötjönen et al., 2010).

Atlas-based segmentation (Hartmann et al., 1999) approach is used for quantification of brain atrophy by considering cerebellum volumes in normal and chronic alcoholics. The technique involves the global transformation of an atlas volume, with the objective to bring it to correspondence. The deformation field calculated on the binary atlas volume creates intradural and cerebellum masks to segment the cerebellum structure. The similarity is assessed by kappa statistics between the manually delineated and automatically delineated cerebellum structure.

The atlas can be single labeled image or probabilistic atlas (Heckemann et al., 2011). The probabilistic atlas is obtained through non-rigid registration of multi labeled images. Then warping technique is followed after registration, to transfer these labels of the atlas image to the subject image and derive the selected ROI by the segmentation technique. So it can be inferred that the atlas construction and registration methods are critically important for segmentation of images.

Structural segmentation of brain through multi-atlas segmentation (Aljabar et al., 2009) is obtained by transferring multiple numbers of manually delineated atlases to the subject and combine them together for effective segmentation. Selective combination of atlases improves the accuracy of sub-cortical segmentation. In particular, the use of age-based selection atlases adds an advantage to multi-atlas segmentation of older subjects.

The label fusion framework (Sabuncu et al., 2010) is carried out by multiple registrations between test and training images. After the process of registration, the Sum of Squared Difference metric measures the similarity between the registered images, before subjecting it to the segmentation step. It also focuses on hippocampal volume measurement correlating with the age of the person, to predict whether the subject brain is affected with AD or it is Normal. Accurate hippocampal volume measurement by local weighted voting and semilocal weighted fusion shows the better results than global weighted fusion and majority voting. The multiple pair wise registrations and the manipulation of the training data introduces computational complexity in the proposed label fusion algorithms.

The selection of the best atlas (Sanroma et al., 2014) is performed by non rigid registration of atlas images and the computation of ground-truth ratio between the pair of atlas images. It is followed with the computation of pairwise feature for each region between pair of atlas images and finally with the target image. Then the segmentation performance is computed and ranked, to select the best atlas. 


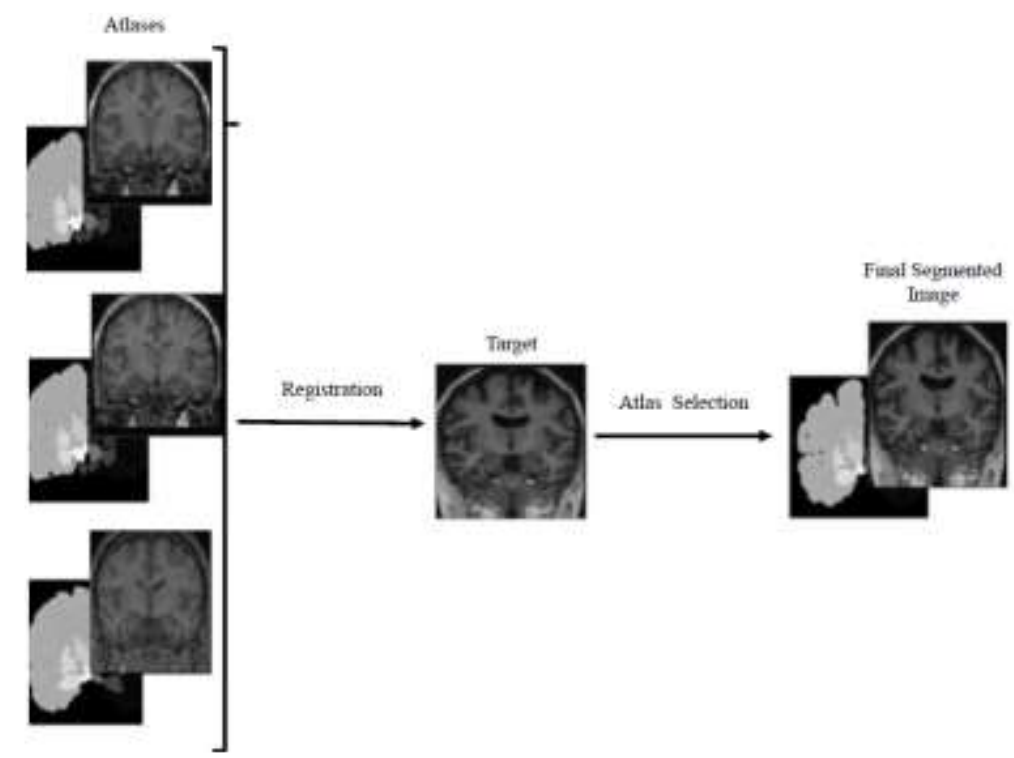

Fig. 3. Atlas Based Segmentation for Alzheimer's disease detection (Lötjönen et al., 2010)

The new labeling techniques (Sdika, 2010) such as Nearest Neighbor transform with Erosion labeling (NNE) and Nearest Neighbor transform based labeling (NN) are proposed, to label complex and folded cortical structure. The quality of automated segmentation techniques are measured by Relative Overlap (RO) and Mean Accuracy (MA) metric and the result indicate improved performance of $\mathrm{NN}$ shown on cortical regions and the increased performance of standard labeling is shown on deep gray structures. The segmentation produced by NN is inaccurate and rough at the border of the structure while NNE labeling is an improvement over NN for the smoother segmentation.

The evaluation of atlas selection strategies (Rohlfing et al., 2004) are very important in atlasbased segmentation. Four different approaches considered for the atlas selection are: Registration to Average Shape Atlas Image (AVG), Registration to an Individual atlas image (IND), and Registration to all images from a database of individual atlas image with the subsequent Multi-Classifier Decision Fusion (MUL) and Registration to most similar image from a database of individual atlas image (SIM). The obtained segmentation results are evaluated by considering mean similarity index between manual and automatic segmented images. Among the four atlas selection strategies, MUL shows an improved performance of segmentation.

Incorporation of statistical model is necessary to select the best atlas for registration (Carmichael et al., 2005). Then the alignment of brain images to the standard atlas is carried out by a fully-deformable method, to aid the process of automated segmentation.
Further the performance of automated segmentation is analyzed by various tools like AIR, SPM and FLIRT (Shenton et al., 1995).

\section{Hippocampus Segmentation}

Hippocampus (HC) is a region located in the medial temporal lobe of the brain and plays a vital role in the process of memory consolidation. So the segmentation of $\mathrm{HC}$ is necessary to study age related problems as shown in the Fig.4. (Carmichael et al., 2005).

Hippocampus is an important biomarker utilized in the detection of various neurodegenerative disorders. The extraction of Gradient Distribution Boundary (GDB) (Zarpalas et al., 2014) is performed on the gray matter tissue and these boundary properties are modeled at each anatomical location to develop gradient based reliability maps. Then by using active contour model, appropriate image information is included for the accurate segmentation of Hippocampus. The increased dice coefficient metric indicates the accuracy of automated segmentation and proves its applicability in manual segmentation protocol. The focus is necessary to introduce bias correction concept and to identify the white matter borders within 3GDB.

Auto Context Model (ACM) (Tu and Bai, 2010; Morra et al., 2008) is a technique proposed for hippocampus segmentation of $\mathrm{T} 1$ weighted MRI images. To acquire global shape information and location of hippocampus, all the brain images are initially registered to the template. During the training phase, manually labeled segmentation learns the rules for classification of hippocampal and non hippocampal region by using modified adaboost method. 


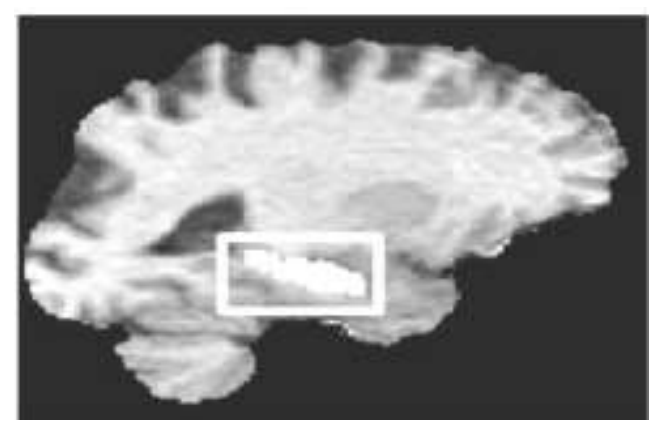

Fig. 4. Hippocampus Segmentation for Alzheimer's Disease Detection (Carmichael et al., 2005)

Then the performance of hippocampus segmentation are evaluated by metrics such as precision, recall, relative overlap, similarity index and hausdorff distance. The ACM segmentation shows less error prone metric when compared to other automated hippocampus segmentation.

The Hippocampal measure is computed by using the techniques of Voxel Based Morphometry and ROI-based approach (Testa et al., 2004). In ROI, measurement of the hippocampal volume is computed on the whole hippocampus by using Mann-Whitney U test (Nachar, 2008). In voxel-based approach, SPM99 is used for computing GM volume which aids in identifying the discriminative region. Both the measurement were assessed in the total group by computing areas under the curve of receiver operating characteristics. Detection of hippocampal atrophy by VBM analysis show better results and ROIbased analysis show the superior performance for measurement of the amygdala and temporal gyri.

Symmetric Normalization (SyN's) (Avants et al., 2008 ) is used in the volume measurements of MRI images. The volume measurement estimated by SYN, is in close association with the volume measurements obtained by experts labeling. Hence it is a valid technique chosen for normalization and anatomical measurements of volumetric MRI in AD patients and also for the elderly individuals who are at risk. In addition to normalization and volume measurement, SyN also provides a dense space-time map and transformation inverses of the subject image.

A learning based wrapper method (Wang et al., 2011) is an effective technique to be utilized after automated segmentation to improve its accuracy. During segmentation, systematic segmentation errors are generated on training data and it is identified by the wrapper method with the help of intensity, spatial and contextual patterns of an image.

Automated algorithm developed for the segmentation is evaluated by Expectation-Maximization algorithm (Warfield et al., 2004) for Simultaneous Truth and Performance Level Estimation (STAPLE) to ensure their suitability for the particular application. It examines the performance analysis of segmentation algorithm by calculating the accuracy and precision.

\section{Registration}

Image registration also known as alignment/coregistration and it is the process of aligning different images of the same subject's follow-up data or same subject with different modality or different subject with the same disease. The target and reference image establish the correspondence between voxels, which aids to fuse information from the images and also to identify the location of the affected region within the diseased individual (Hill et al., 2001). The measure of atrophy over a period of time, which is very important for clinical trials are assessed by Serial MRI Scans Registration. Hence, the atrophy rate is measured by registering the images before and after drug treatment (Fox et al., 2000). Registration techniques suitable for multimodal images are discussed in later sections.

An automated Mutual Information Registration (Wells et al., 1996; Maes et al., 1997) for multi-modal images is largely data independent and does not require preprocessing. Mutual Information Registration is affected by factors such as in homogeneity, geometric distortion and noise.

The Fluid registration (Bro-Nielsen and Gramkow, 1996) is performed on longitudinal MRI images, to compute the deformation field occurring at each voxel. The deformation field computed for an entire image is measured by a jacobian value. Voxel for expansion and voxel for contraction are the two images created based on the jacobian value. Then a group analysis is performed by SPM, to find the expansion of lateral and third ventricle in mildly affected $\mathrm{AD}$ and expansion of perihippocampal region in moderately affected AD. The contraction is observed in the posterior part of the cingulate gyrus, the inferior and lateral parts of the temporal lobes (Scahill et al., 2002).

A nonlinear diffusion filter (Rexilius et al., 2001) is used to obtain appropriate feature points, required for an initial sparse estimate of the deformation field. Normalized Cross-Correlation (NCC) is a similarity measure used for finding the correspondence between reference and template volume. In the next step, the volumetric deformation across the image is inferred from nonrigid registration using an elastic model.

Elastically deforming three-dimensional atlas (Gee et al., 1993) locates the similar anatomical brain volumes across MRI images in the data set. Features used for matching are brain surface, ventricles, edges of cortical, subcortical structure, gray and white matter boundary. It performs well for larger and regular structure, but its performance is low for smaller and irregular structure. The accuracy of each match is evaluated by the amount of overlap between the deformed atlas and elastically mapped atlas. Future focus is to develop automated technique for preprocessing of images required for elastic registration. 
The Spatial Transformation Algorithm for Registration (STAR) (Friston et al., 1995; Davatzikos, 1998) is proposed to capture subtle morphological characteristics of an individual brain. As it is a featurebased approach, it utilizes the structural information such as outer cortical surface, the ventricular surface and the sulci. A map is formed between homologous features of an individual brain and the target brain to drive a three-dimensional elastic warping transformation. The advantage of this technique is that information present in the original, non-normalized images is preserved even after normalization.

The optimum template selection method (Wu et al., 2007) chooses the best template from a family of templates by using the parameters such as normalized mutual information and ROI. These parameters give best local registration accuracy for a selected template. The template selection is very important for highly varying cortical structure. The selection of multiple templates required for atlas-based segmentation produced significantly better Overlap Ratios (ORs) and more reliable volume estimates. Then Intraclass Correlation Coefficients (ICCs) is calculated to measure the difference between the manual tracings and the automated labeled results.

Based on age, the local deformation of the image varies significantly across patients. The local deformation cannot be described properly by the parameterized transformation. Hence, 3-D deformable objects is modeled by a Bspline based Free Form Deformation (FFD) model (Rueckert et al., 1999). Then the similarity measures such as Squared Sum of Intensity Differences (SSD) and Correlation Coefficient (CC) are used to measure the registration quality across the different transformation models considered. The performance of Nonrigid registration (Crum et al., 2014) can be improved by increasing the resolution of mesh in spline based FFD.

Rapid Alignment of Brains by Building Intermediate Templates (RABBIT) (Tang et al., 2009), develops a statistical deformation model by acquiring knowledge from the deformation field of the training sample. The development of intermediate template similar to the brain under registration is produced by the calculation and optimization of the parameters of intermediate deformation field. Hence RABBIT has potential to attain rapid and accurate deformable image registration. Therefore the speed of RABBIT is five times more than HAMMER. The high registration accuracy by HAMMER can be obtained through refinement of the intermediate templates.

An automated Reverse Brain Masking (RBM) (Keihaninejad et al., 2010) measures the IntraCranial Volume (ICV). ICV is required for normalizing the regional volumes of brain. RBM uses the sum of three tissue probability maps to estimate the ICV probabilistic mask in MNI standard space. Then Intra Class
Correlation Coefficient (ICC) is utilized to measure the similarity between manual and RBM based ICV measurement methods. The RBM shows similar performance to manual ICV measurement on both 1.5T and 3T scanner and achieves higher accuracy on both normal and AD subjects. The conventional methods such as SPM and FSL are not consistent in ICV measurement across different scanners but RBM is more consistent and reliable.

Brain warping techniques such as Large Deformation Diffeomorphic Metric Mapping (LDDMM) (Tang et al., 2014), characterizes the variations in the brain regions due to neurodegenerative disease, either by the volume or shape analysis. It is important to detect distinct subregions of hippocampus (3 Subregions- CA2, CA3 and Dentate Gyrus) and amygdala (4 subregions- Basolateral, Basomedial, Centromedial and Lateral nucleus) from high-resolution images. This aids in understanding the disease pattern associated with these structures. The initial momentum vectors from LDDMM are subjected to Principal Component Analysis (PCA). The Principal Components (PC) obtained for each structure is subjected to Linear Discriminant Analysis (LDA), to identify the associated biomarker in AD or MCI. The results obtained from this method show mild regional atrophy of basal ganglia structures, globus pallidus and atrophy in the vertices of the surfaces of the thalamus for both MCI and AD groups. Mild expansion in caudate nucleus has resulted in subjects only with AD.

\section{Morphometry}

Morphometry is the technique applied to find the local and global anatomical differences existing in the affected brain. This anatomical difference can be detected by subjecting the brain to different types of morphometric techniques such as Voxel Based Morphometry, Deformation-Based Morphometry, Shape Based Morphometry and Feature Based Morphometry. A brief discussion of these morphometric techniques are explained in further paragraphs and Table 3 also includes the advantages and disadvantages of some of the techniques.

\section{Deformation Based Morphometry}

A Deformation field morphometry is a technique based on the nonlinear transformation of source image onto the reference brain. The calculation of deformation field determines the shape of the human brain at the voxel level. And the local volumetric differences between the source and the reference image are obtained by the calculation of Local Volume Ratio (LVR) (Pieperhoff et al., 2008). LVR map is generated by the combined LVRs of all the voxels in an image. The LVR maps of different source images are compared to infer the volumetric changes in the brain. A unified statistical framework using 3D displacement field vector (Chung et al., 2001) was proposed to measure the displacement and volume 
changes of the brain due to morphological changes observed in the diseased state. The rate of Jacobian change and local volume change at each voxel can be calculated, to measure growth or loss of the brain tissue.

\section{Voxel Based Morphometry}

Voxel-Based Morphometry (VBM) (Whitwell, 2009) identifies the atrophy of brain among the group of subjects affected with $\mathrm{AD}$, through the statistical methods. This automated technique concentrates more on gray matter and indicates the region with gray matter loss is caused due to $\mathrm{AD}$. Improvised results can be observed with Family Wise Error (FWE) correction and lenient False Discovery Rate (FDR). FWE improves the result for the sample with larger size and FDR is suitable for the smaller size. But it cannot provide reliable information for an individual subject.

SPM99 package (Ashburner and Friston, 2000) is used to perform voxel-based morphometry, with more emphasis on segmenting the gray matter from the MR images with non-uniformity artifact. The segmented gray matter is smoothened using the gaussian kernel. Then the statistical tests are performed on the smoothened image to perform the comparison among different groups.

Voxel based morphometry (Senjem et al., 2005; Mechelli et al., 2005) infers the structural differences for a group of subjects by using the gray and white matter volumes. It processes the entire brain, with the consideration of all the voxels, followed by the statistical test for analysis. Hence it finds its application in detection of various diseases such as AD, Schizophrenia, Parkinson's Disease, Bipolar Disorder, Autism and also the enlargement of certain brain regions influenced for the healthy normal's through the learning process. The main difficulty is in spatial normalization of a typical brain and images have to be considered from the same scanner and common parameters for the calculation.

The tissue density increases during contraction of an image due to the atrophy of the brain. Increment or decrement in the tissue density is directly related to the volume of its respective structure. So the regional volume measurements and comparison are performed using RAVENS (Regional Analysis of Volumes Examined in the Normalized Space) (Davatzikos et al., 2001) density map generated for each tissue of interest. Voxel based morphometry performed by RAVENS is better than Statistical Parametric Mapping (SPM).

A voxel-based discriminative map (Wang et al., 2009) aids in AD classification. The average brain atlas is considered as a probability map, calculated for both $\mathrm{NC}$ and $\mathrm{AD}$ subjects. The discriminative map is obtained by subtracting the probability map of an healthy control and patients with AD. Then the matching coefficient are calculated between the obtained discriminate map and the training input. Based on the calculation, test input is considered as a patient if matching coefficient value is more than the cut-off point of training subjects. It is an alternative method to volume-based classification technique.

Voxel based morphometry detection cluster extracts the features like mean, standard deviation and voxel intensities from the GM volume. And these features are subjected to classification by using linear, non linear and the diverse adaBoost SVM (Li et al., 2005). Diverse Adaboost SVM is found to perform better than linear and non linear SVM. This method also resulted in higher sensitivity results for $\mathrm{AD}$ classification. Focus is necessary on feature extraction from deformation based morphometry (Savio et al., 2009).

The prognosis of MCI to $\mathrm{AD}$ uses MRI and CSF biomarker for pattern classification (Davatzikos et al., 2011). The method involves tissue segmentation and development of RAVENS MAP by using high dimensional image warping technique (Chen and Herskovits, 2010). Then t-statistic is used to find the group differences among the considered subject images. In addition to gray matter atrophy, white matter atrophy is also observed around the hippocampus and other temporal lobe structures. The inclusion of WM atrophy provides more pronounced results in early identification of MCI individuals who are likely to convert to AD. Even Spatial Pattern of Abnormalities for Recognition of Early AD (SPARE-AD) score (Davatzikos et al., 2009) is used for calculating the abnormality score for the subject with MCI. The positive score of SPARE-AD indicate $\mathrm{AD}$ and negative indicate Normal control, hence SPARE-AD score, morphological measurements and CSF biomarker are considered as good predictors in determining the future progression of MCI.

\section{Feature Based Morphometry}

Feature-based morphometry (Zhu et al., 2016; Niyogi, 2004) aims to identify distinctive anatomical patterns of a subset of subjects due to disease as shown in Fig. 5. (Diaz et al., 2010). Localized image features are extracted and these features are analyzed at the characteristic level. It fetches both local and global topological information from the data. The probabilistic model is applied to the extracted image features to relate them to a particular group based on the anatomical pattern. Hence it can be used as a biomarker for the classification of $\mathrm{AD}$. The discriminative feature selection is based on Fischer criterion and obtained features are noise resistant. Capturing of difficult patterns from the $\mathrm{AD}$-affected brain images can be improved by utilizing non linear modeling. 
Vinutha N. et al. / Neuroscience International 2016, 7 (2): 19.44 DOI: 10.3844/amjnsp.2016.19.44

Table 3. Feature based morphometry techniques for Alzheimer's disease detection

\begin{tabular}{|c|c|c|c|}
\hline Authors & Algorithm & Advantage & Disadvantage \\
\hline$\overline{\text { Liu } \text { et al. (2015) }}$ & Stack Autoencoders & $\begin{array}{l}\text { High level features improves } \\
\text { accuracy and sensitivity }\end{array}$ & $\begin{array}{l}\text { Low accuracy on } \\
\text { testing set }\end{array}$ \\
\hline Bron et al. (2015) & Feature selection & $\begin{array}{l}\text { Wrapper and filter methods } \\
\text { improves the performance }\end{array}$ & $\begin{array}{l}\text { Trade off between area under } \\
\text { curve and complexity are criteria } \\
\text { in feature selection }\end{array}$ \\
\hline Cheng et al. (2015) & $\begin{array}{l}\text { Feature selection, sample } \\
\text { selection and classification }\end{array}$ & $\begin{array}{l}\text { Improves the sensitivity, } \\
\text { accuracy aids in predicting } \\
\text { MCI conversion }\end{array}$ & $\begin{array}{l}\text { Improvement of prediction by } \\
\text { including auxillary domain data }\end{array}$ \\
\hline Zhu et al. (2016) & $\begin{array}{l}\text { Canonical Correlation } \\
\text { Analyis (CCA) }\end{array}$ & $\begin{array}{l}\text { Improves the } \\
\text { accuracy }\end{array}$ & $\begin{array}{l}\text { More than two modalities } \\
\text { to be focused }\end{array}$ \\
\hline Hu et al. (2016) & $\begin{array}{l}\text { Wavelet frame, linear } \\
\text { SVM for classification }\end{array}$ & $\begin{array}{l}\text { Discriminates MCIc } \\
\text { and MCInc }\end{array}$ & $\begin{array}{l}\text { Focus on feature } \\
\text { selection }\end{array}$ \\
\hline
\end{tabular}

An increased cortical thickness (San et al., 2016) was found to be effective when both education and physical exercise were combined. Increased cortical thickness in prefrontal cortex, precuneus, left postcentral gyrus, inferior parietal region correlates with longer duration of exercise but not with the intensity of exercise.

A supervised method of training Artificial Neural Network (ANN) (Jain et al., 1996; Cárdenas-Peña et al., 2016) is proposed for computer aided diagnosis. The classification of dementia is very important because Mild Cognitive Impairment (MCI) is the intermediate stage between $\mathrm{NC}$ and $\mathrm{AD}$, and it may or may not progress to AD. The features extracted from MRI scan of brain are volume, surface area and thickness. Affinity between this projected feature space and class label can be increased by the inclusion of Centered Kernel Alignment (CKA) and over-fitting is avoided by including the large number of subset of samples.

A feature representation through deep learning is applied for brain disease diagnosis and prognosis. The main motivation is to extract high-level information hidden in the low-level features with the help of Stack Auto-Encoder (SAE) (Vincent et al., 2010; Suk et al., 2015). To extract high level information, it is necessary to integrate both low-level and latent features to generate the augmented feature vector. Then the sparse learning is applied on this augmented feature vector, for efficient selection of features and to label the subjects into different stages of the disease. Finally features from different modalities are combined through Multi Kernel SVM. Diagnostic accuracy is improved further by the fusion of various features from different modalities.

Global Gray Matter (GM) atrophy is recognized as a marker in $\mathrm{AD}$ or MCI progression. During clinical trials, atrophy of the entire brain are measured commonly by Structural Image Evaluation using Normalization of Atrophy (SIENA) (Smith et al., 2004) and Brain Boundary Shift Integral (BBSI) (Fox and Freeborough, 1997). The proposed method also determines the Global atrophy by integrating Non-linear registration with Jacobian values (Anderson et al., 2012). The results inferred show that, statistical power of the proposed method is similar to BBSI, SIENA, but it is more than segmentation and subtraction of serial GM volumes.

An automated correspondence detection algorithm i.e., Wavelet Based Attribute Vector (WAV) (Kumar et al., 2009), efficiently captures the anatomical features of each voxel from the original MR brain images and hence it can be used as a morphological signature for each voxel. Three matching techniques of WAV: (i) using WAV similarity (ii) matching using statistical models of WAV's and (iii) matching by incorporating shape constraints, are required to determine the correspondence between the images, by finding the similarity between the attribute vectors. The performance of the algorithm is equivalent to an expert with the incorporation of the shape constraint and it can be improved further by incorporating prior knowledge of spatial relationship of voxels (Xue et al., 2004).

Multiple features extracted from Magnetic Resonance Imaging (MRI), Cerebrospinal Fluid (CSF), Neuropsychological and Functional Measures (NMs) aid in the prognosis of MCI (Cui et al., 2011). The minimum Redundancy and Maximum Relevance (mRMR) filter (Akadi et al., 2009) assign the ranks to the extracted features. Then the subset of features is subjected to Lib SVM for classification. The combination of MRI, CSF and NM results in high sensitivity and it is also used for calculating the conversion time from MCI to AD using the predictive values.

Large Deformation Diffeomorphic Metric Mapping (LDDMM) (Qiu et al., 2009) determines a surface deformation map, to estimate the deformation from the template to the surface of each subject. With reference to the template, Outward-deformation of subject's structure is marked with positive value and inward-deformation of subject's structure is marked with negative value. Based on the geometry of each structure, the random fields are calculated and it is followed by modeling to find the inter-relationship among the brain regions (Wold et al., 1987). Then the principal components determine the group differences among $\mathrm{AD}, \mathrm{MCI}$ and $\mathrm{NC}$ 


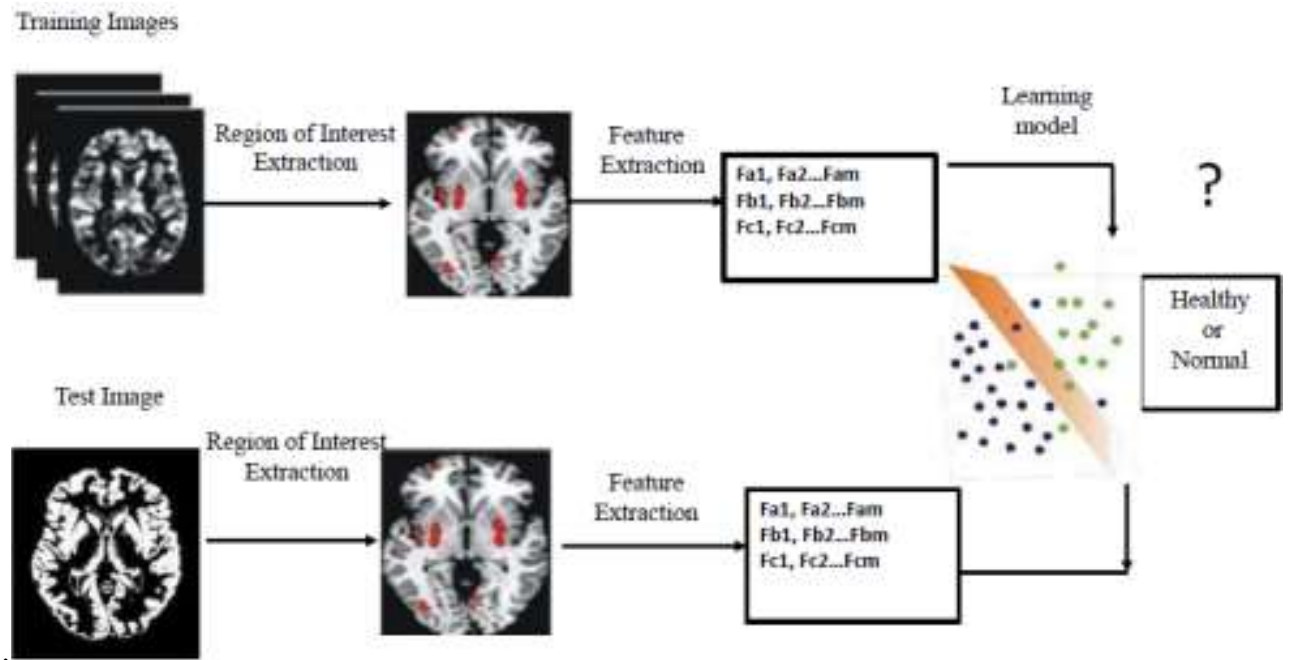

Fig. 5. Feature based morphometry for Alzheimer's Disease detection (Diaz et al., 2010)

The deep learning architecture is used in the classification of four different stages of AD such as Normal Control, cMCI (Mild Cognitive Impairment Convertor), ncMCI (Mild Cognitive Impairment Non Convertor) and AD. This technique helps in diagnosis of a patient at particular stage by using either single modal or multi modal data. The gray matter volume and the Cerebral Metabolic Rate of Glucose (CMRGlc) are the features extracted from MRI and PET images. These extracted features are unlabeled and trained to the auto encoder to fuse the multimodal data and to learn high level features, even in the absence of one modality. The high level feature obtained is subjected to Softmax Regressor, to generate the label for a particular stage. Then it is followed by the calculation of stability score. The score aids to identify the region with highest score as an important biomarker and also to recognize the progressed state of the disease. It works better on large datasets and finds synergy between different biomarkers used. Accuracy on training set is better than test set (Liu et al., 2015).

A robust deep learning system utilizes drop out technique ( $\mathrm{Li}$ et al., 2015), which is an improvement over the classical deep learning. CSF biomarkers and volumetric features from MRI and PET images are subjected to PCA to reduce the dimensionality of the extracted features followed by stability selection technique. So classical deep learning with drop out performed better than without using dropout technique for the small dataset. It is necessary to use Multi Kernel SVM for diagnosis of AD patients.

A denoising auto-encoder denoises the corrupted input and fetches the high level features like edges in an unsupervised way. This builds the relationship between denoised input and higher level features to progress the performance of the SVM classifier (Vincent et al., 2010).

The direct approach (filter) and an iterative approach (wrapper) are the two novel feature selection methods (Bron et al., 2015) proposed based on p-maps. The trade- off between Area Under Curve and Complexity are the criteria involved in choosing best feature selection method. Both the filter and wrapper approach perform better than SVM weight vector.

The Domain Transfer Feature Selection (DTFS), Domain Transfer Sample Selection (DTSS), Domain Transfer Support Vector Classification are the techniques used in the classification of MCI (Cheng et al., 2015). DTFS method utilizes auxiliary domain (i.e., AD, Normal Control) and learns about target domain (i.e., Mild Cognitive Impairment, Non Mild Cognitive Impairment). The subset of features of both auxillary and target domain are extracted from multimodal data and subjected to DTSS feature selection, to develop cross domain kernel matrix for MRI, PET and CSF biomarkers. The proposed technique improves sensitivity, accuracy and area under curve, thereby correlating to the improved prediction of the MCI conversion. Further improvements can be obtained by including auxillary domain data.

Changes in hippocampus, ventricles and whole brain are sensitive to $\mathrm{AD}$ progression but these regions are also sensitive even in healthy aging adults. The results indicate Multi- Temporal Lobe (MTL) cortical Regions in particular Entorhinal cortex is sensitive to the early stage of AD. Mixed effect regression model determines longitudinal volumetric change as an outcome measure to detect the regions, which are sensitive to drug effects in MCI. The results derived from the regression model shows that, drug treatment is effective in global and subregional MTL and cortical region (Holland et al., 2009).

The statistical classification method (Fan et al., 2005) identifies the abnormality of the brain, by aggregating regional morphological features with common classification strategy through high dimensional template warping. These features are classified through Support Vector Machine-Recursive Feature Elimination (SVMRFE) classifier (Lin et al., 2012). This classifier performs both adaptive regional feature extraction and 
recursive feature elimination, to retain the most relevant feature for classification. The need for prior information is eliminated by adaptive regional feature extraction technique and it results in high classification rate.

Multivariate Examination (Fan et al., 2007) of brain abnormality, involves regional statistical feature extraction from the voxel wise morphometric and functional representation. The extracted discriminative features are subjected to hybrid feature selection and nonlinear SVM classifier. This process is carried out to identify the abnormalities of the brain caused by the prenatal cocaine exposure. Simultaneous extraction and selection of features from both functional and structural MR images result in increased classification accuracy.

Multi-Kernel learning framework (Liu et al., 2014; Joshi et al., 2010) is proposed for combining their multimodal features such as Cerebrospinal Fluid (CSF) biomarkers and Magnetic Resonance Imaging (MRI) Left Hemisphere Hippocampus shape, Right Hemisphere Hippocampus shape and ROI. Group sparsity among different image modalities and complementary information among different kernels are obtained by using Random Fourier Feature and L2, 1 norm regularization. Improved results are noted with the following parameters like specificity, accuracy, matthew's correlation coefficient and area under ROC curve. This aids in identifying the important regions closely related to AD. The significant regions identified are hippocampal formation right, hippocampal formation left, amygdala left, precuneus right, lateral ventricle right.

The classification of AD/MCI is proposed by Inherent Structure-based Multiview Learning (Liu et al., 2015). Among the three tissues, GM tissue provides more information in detecting $\mathrm{AD}$. This technique utilizes pre-defined template library and these templates are subjected to affinity propagation for obtaining a high representative data points (exemplars). Each subject to be classified, is nonlinearly registered to the multiple templates to obtain multi-view features. Then it is followed by multitask feature selection to fetch informative features on each view and subject it to Multiple Support Vector Machine (SVM) classification. Multi-class classification between AD vs Normal Control (NC), pMCI (Progressive Mild Cognitive Impairment) vs Normal, pMCI vs Stable MCI is performed. Improved performance is evaluated by considering different metrics such as classification accuracy, sensitivity, specificity, balanced accuracy, area under the receiver operating characteristics curve. To perform a direct comparison of a subject with two different templates, it is necessary that the templates have to be registered to a common space, followed region of interest partition. The classification accuracy can be further improved by adopting different encoding strategy involved in relabeling the subclasses.

An automatic ideal Mid-Sagittal Plane (iMSP) extraction algorithm (Liu et al., 2004) identifies the potential discriminative feature present in the ROI, by extracting both statistical and textural features. Along with the intensity features, the shape features are also included to provide complementary information required for the classification. The highly discriminative features are ranked based on the calculation of Augmented Variance Ratio (AVR). This is followed by Linear Discriminant Analysis (LDA) (Li and Yuan, 2005) a feature subset selection algorithm, which is used as the evaluation classifier.

Feature extracted from deformation and tensor field are $3 \mathrm{D}$ vector field that is split into seven scalar fields XYZ components, theta, phi angles, the determinant of a jacobian matrix. Along with the deformation and tensor field, four levels of voxel neighborhood are also considered as the features. It is followed by statistical parameter calculation for each neighborhood that includes minimum, maximum, mean, variance, 3rd and 4th moments. The selection of feature as a biomarker for the classification of disease is performed by the calculation of augmented variance ratio. The augmented variance ratio searches for the discriminative feature subspaces and corresponding anatomical regions that aids in AD prediction (Liu et al., 2007).

Computer-aided diagnosis of $\mathrm{AD}$ is performed to identify the region which is distinguishable in Normal and AD patients by using two multivariate approaches (Segovia et al., 2012). The first approach utilizes Gaussian Mixture Model (GMM) for modeling the ROI. The number of features obtained is equal to the number of Gaussians, where each Gaussian is varied by its height and density. The feature reduction is achieved by choosing the Gaussian of higher heights that corresponds to be a region of differentiation. GMM achieves better classification by subjecting the obtained features to the linear and nonlinear classifier. Partial Least Square (PLS) is incorporated as a second approach which comprises regression, dimensionality reduction and classification tasks for AD.

Multi-modal and Multi-task learning (Zhang et al., 2012) predicts multiple variables from the multi-modal data that are required for the classification of $\mathrm{AD}$. Both clinical and categorical variables (multi-modal data) are subjected to multi-task feature selection to identify the common subset of relevant features related to a disease. Fusion of the selected variables from multi-modal data is performed by multi-modal support vector machine. The results obtained by multi-modal and multi-task learning techniques, are evaluated by root mean square prediction error metric, that indicates improved performance over the traditional methods.

The two different modalities such as MRI, PET and Clinical score such as ADAS-Cog and MMSE are subjected to Canonical Correlation Analysis (CCA), for Joint Clinical Scores Regression and Multiclass AD status Identification (JRMI) (Zhu et al., 2016). The performance is evaluated by the Correlation Coefficient (CC) and Root Mean Squared Error (RMSE) metric in both Regression and Classification tasks. More than two Modalities can be considered for further improvement. 
Different Biomarkers for $\mathrm{AD}$ diagnosis are CSF measures, MRI morphometry measures (volume of the hippocampus, entorhinal and retrosplenial cortical thickness) and FDG-PET measures (retrosplenial, entorhinal, and lateral orbitofrontal metabolism). These features are subjected to logistic regression analysis, to find good predictors. The combination of CSF biomarkers and MRI morphometry, improved diagnostic classification accuracy and combination of MRI morphometry and FDG-PET give satisfactory results in predicting a clinical decline in MCI (Walhovd et al., 2010).

The wavelet-based feature extraction (Chaplot et al., 2006) aids in the classification of MRI images as normal or abnormal. Representation of MRI images at various resolutions is obtained by the decomposition of a signal into hierarchical scale by wavelet analysis. The extracted wavelet features are subjected to Self-Organizing Maps (SOM) and SVM classifier. SOM is an unsupervised method that generates the same output for similar input pattern. While the SVM classifier uses linear, polynomial and nonlinear kernel for two-fold classification. The performance of SOM (Van Hulle, 2012) and SVM are evaluated by their classification accuracy. The accuracy of classifying normal and abnormal images is high with polynomial and nonlinear SVM compared to SOM.

The Single positron Emission Tomography (SPECT) images are spatially normalized to a template. This enables different images to refer to the same anatomical location. The experts labeled normalized images with four labels, representing different stages of $\mathrm{AD}$. The extraction of latent vectors from this labeled image is obtained through Partial Least Square (PLS) by considering both the variance and class labels of the subjected image. Random Forests (RF), an ensemble of decision trees (Vibha et al., 2006), bagging and majority voting is applied on the extracted latent vectors, to reduce the dimensionality and also to aid in the classification of AD. Generalization error induced by the classifier is minimized by including more number of trees in the random forest (Ramírez et al., 2010).

A wavelet frame (Hu et al., 2016) is a multi-scale image representation approach. Features are extracted from the whole gray matter or gray matter images of hippocampus region for differen scales and direction. The extracted features are subjected to SVM classification to discriminate MCI converters and MCI nonconvertors. Feature selection algorithms are necessary to obtain the redundant information for better classification.

Dual tree complex wavelet transform is applied on MRI data, to simultaneously obtain a class of new features. The new features include scale, directionality and potentially local information based on the magnitude of complex wavelet coefficients. Linear support vector machine is used to classify the subjects suffering from Multiple Sclerosis. High variations in decoding accuracy are observed for different scales and show the superiority for the scale with low-frequency information (Hackmack et al., 2012).
Feature extraction by Two-dimensional Discrete Wavelet Transform (2DWT) (Zhang et al., 2011; Joshi et al., 2010) decomposes the images into three levels. At the third level, Harr wavelet feature is extracted. The feature vector is subjected to normalization and reduction in the dimension of feature vector through PCA. The images are then classified as normal or abnormal by subjecting the reduced features into back propagation Neural Network.

After registration using hammer, 93 manually labeled ROI are obtained. The volume of ROI is extracted from MRI images; the average intensity of the same ROI is derived from the affine registration of PET image. Along with volume and average intensity, CSF biomarker is also considered as a feature. The multiple-kernel SVM (Zhang et al., 2009) integrates the features from multiple modalities of biomarkers to classify AD or MCI from healthy controls. The proposed technique results in high classification accuracy and high sensitivity for MCI classification of subjects (Zhang et al., 2011).

Using Freesurfer and Morphobox tool, Volume based Morphometry (VolBM) are computed to the diagnosis of AD. As the first step in morphobox tool, labeling of the Total Intracranial Volume (TIV) voxels within the brain tissue is performed even without the use of prior information. In the next step, structural segmentation is performed by combining tissue maps with anatomical masks taken from a single subject template through nonrigid registration, whereas in the case of free surfer both these steps are combined. Hence computation time in free surfer is more compared to morphobox tool. Multivariate volume-based classification using freesurfer and morphobox tool performed better than whole brain voxel based morphometry determined by SPM (Schmitter et al., 2015).

Fully automatic intensity based inter-subject image registration involves global affine registration to a reference image followed by local affine transformation within volume of interest. After the registration step, the transformed image is subjected to PCA. PCA is a statistical technique, that linearly transforms the data into principal components, that helps to identify the patterns of variation among the subjects (Webb et al., 1999).

Using only the neuropsychological measure, it is impossible to determine differences among the prodromal stage of AD and healthy aging. But including multiple biomarkers like MRI, PET, SPECT improves the prediction of different stages of Alzheimer's Disease (Nestor et al., 2004; Joshi et al., 2010).

The macroscopical anatomical differences between the brains of the different population are to be identified with the following steps. The procedure involves, mapping of the subject image into stereotactic space through spatial normalization of the brain. In the next step, normalized image is subjected to Multivariate Analysis of Covariance (MANCOVA) and Canonical Variates Analysis (CVA), 
that helps to characterize the anatomical difference between the subject images (Ashburner et al., 1998).

Volumetric texture features have better discriminative power than the $2 \mathrm{D}$ texture obtained for the slice data. So the features calculated for the volumetric data are Entropy, Energy, Contrast, Homogeneity, Sum Mean, Variance, Correlation, Maximum Probability, Inverse Difference Moment and Cooccurrence Matrices. Co-occurrence Matrices provide more information in the identification of the texture within an organ. Then the extracted volumetric texture descriptors are subjected to robust classifiers for the classification of AD or Normal (Kurani et al., 2004).

The Spatial Gray-Level Dependence Method (SGLDM) (Freeborough and Fox, 1998) is used for the quantification of texture over the coronal slices of the brain region. Textural features obtained for different angles and different scales are subjected to the sequential forward selection method to derive the subset of features, based on the Mahalanobis distance between the control and patient groups. The extracted textural features are discriminative in AD and Normal control subjects.

\section{Shape Based Morphometry}

The shape based statistical analysis (Golland et al., 2001), constitutes a warping technique to extract the shape parameters/shape descriptors (Fourier descriptors, spherical harmonic functions) and non-parametric models (landmark based descriptors, distance transforms and deformation field). Kernel-based classification technique (SVMs) is applied to analyze the shape differences present in the anatomical structure of the abnormal brain are shown in Fig. 6. (Kim et al., 2015). The main advantage of this method is to establish a link from the feature space to original shapes.

The two different shape representation schemes are (i) A fine scale, global surface characterization using spherical harmonics (ii) A coarsely sampled medial representation (3D skeleton). The shape fetches the information of structural similarity or dissimilarity, which could not attain through volume analysis. The local and global parameters such as length, elongation, bending, width, complexity, bumpiness explains the pathological changes observed in neurodegeneration (Gerig et al., 2001).

Direct Hippocampal Mapping (Costafreda et al., 2011), models the intrinsic geometric properties of hippocampus to find the variations across the subjects. The intrinsic radial distance obtained for hippocampus in each subject was normalized with global head size effects. Then general linear modelling examines the effects of changes in clinical scores i.e., Mini-Mental State Examination (MMSE) and Consortium to Establish a Registry for Alzheimer's Diseasecognitive battery (CERAD recall) on normalized hippocampal volume and radial distance. The result infers that bilateral focal hippocampal atrophy is associated with subsequent decline in CERAD scores, while right hippocampal atrophy was linked to subsequent deterioration in MMSE scores.

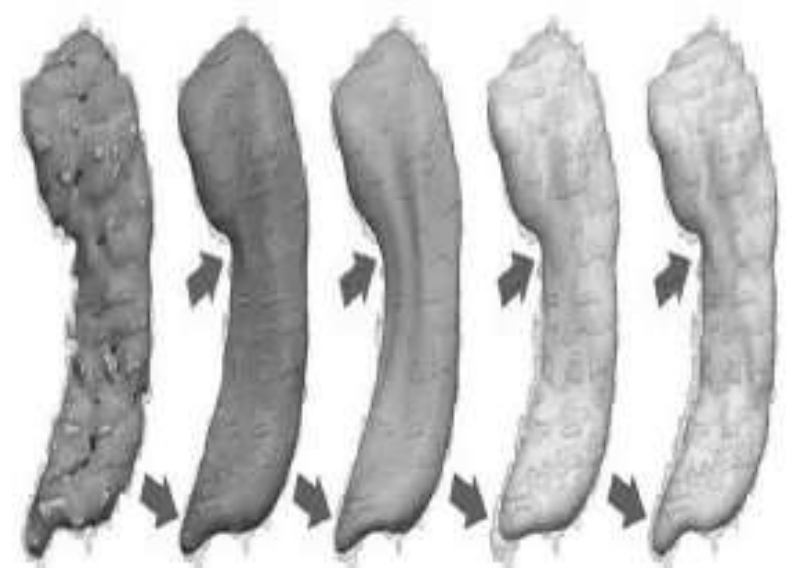

Fig. 6. Shape based morphometry for Alzheimer's disease detection (Kim et al., 2015)

MRI images of elderly subjects affected with AD are evaluated by creating maps of the hippocampal and ventricular structure. As the disease progresses, the change in ventricular and hippocampal volume is observed over a period of time. The map of radial atrophy is obtained for each hippocampus by calculating radial distances from homologous hippocampal surface points to the central core of the individual's hippocampal surface model. Similar modeling steps are applied to temporal horns of the lateral ventricles. The statistical maps obtained for radial hippocampal distance and ventricular expansion, shows the local differences are existing among the structures over a period of time. The distribution of features in statistical maps is measured by permutation methods. After the measurement, it is inferred that, the maps of ventricular radial distance and left hemisphere of hippocampus link with follow up MMSE scores. The intra-rater and inter-rater reliability for the hippocampal and temporal horn measure asses the reliability of measures based on manual outlining (Thompson et al., 2004).

A shape-based classification approach quantifies the shape differences of the anatomical structure caused due to a disorder. A snake approach computes fixed topology skeleton to represent the shape of a region to be differentiated. The topology determines the images to be classified under schizophrenia or normal, by adopting two different linear classification techniques: the fisher linear discriminant and the linear support vectors (Golland et al., 1999).

\section{Classification}

The extracted features or the anatomical patterns from the brain images are necessary to distinguish 
them into normal or affected with AD. The performance of different classification techniques are evaluated by considering the metrics such as accuracy, sensitivity and specificity. The following paragraphs and Table 4 discuss about the classification techniques with their limitations and advantages.

The accuracy of Automated Computer Classification Technique (ACC) is evaluated by considering varying acquisition parameters and image quality. This technique extracts Jacobian Determinants (JD) (Teipel et al., 2007) from dense deformation field and also extract Scaled Gray Level Intensity (SGLI) from the volume of interest. The JD and SGLI are more robust to varying acquisition parameter and image quality (Duchesne et al., 2008).

In the absence of diagnostic information, imagebased biomarkers are extracted by semi-supervised pattern classification (Filipovych et al., 2011). This approach uses the unlabeled images of MCI and labeled images of normal and AD. Then Leave One Out (LOO) semi-supervised classification detects the structural patterns that separate AD from Normal and final label is obtained for the subject of MCI through the voting procedure.

The classification of $\mathrm{AD}$ is improved by the inclusion of MCI subjects from the multimodal data (Zhang and Shen, 2011). In the objective function of Multimodal Laplacian Regularized Least Squares (mLapRLS), AD and Healthy Control (HC) are used for supervised learning. MCI together with $\mathrm{AD}$ and $\mathrm{HC}$ is used for unsupervised learning to inherent geometric structure of whole data. The accuracy, sensitivity, specificity can be improved further by considering the MCI subjects.

Using the SPECT images, the classification of Alzheimer's Disease is proposed by considering voxel intensities as a feature. The amount of samples used for classification are smaller than the dimensionality of an image, hence images are subsampled to minimize both dimension and computation time. Even without specific knowledge to pathology, PseudoFischer linear Discriminant Classifier (PFLDC) shows improved performance (Stoeckel et al., 2004).

Maani et al. (2014) proposed volumetric texture classification by computing local gradients in a $2 \mathrm{D}$ plane and 3D volume. Developing 2D and 3D kernels improve the speed of local gradient computation and also to generate the textural features. The texture pattern developed by this technique is robust to imaging artifacts and helps in evaluating tissue classification problem, to distinguish between old and young brains. In the presence of gaussian noise, it performs better than sobel and first order derivative of gaussian and it is more robust to blurriness.

The multiple features such as volume of tissue, 2D and 3D ventricular shape are extracted. MannWhitney $U$ test removes the low discriminative features with low discriminative power and the dimensionality of the features is reduced further by PCA technique. A hybrid of Particle Swarm Optimization (PSO)-SVM improved the classification, with the increase in accuracy, sensitivity and specificity (Yang et al., 2013).

The classification of individual images is based on categorization of a similar pattern to a particular group, obtained by calculation of Brain Morphological Signature (BMS). RAVENS a mass preserving framework accomplish the shape transformation technique to obtain ravens map by warping a subject image to the template and after transformation the tissue of different regions of the brain are preserved. A Ravens map is represented in a hierarchical way through wavelet decomposition, with the objective of minimizing the dimensionality of the image. Features derived from different tissues are integrated to obtain BMS, and it is subjected to nonlinear SVM classifier. By using Nonlinear SVM classifier, complex patterns of morphological group differences are identified among the subject images (Lao et al., 2004).

Table 4. Classification techniques for Alzheimer's disease detection

\begin{tabular}{|c|c|c|c|}
\hline Authors & Algorithm & Advantage & Disadvantage \\
\hline Maani et al. (2014) & $\begin{array}{l}\text { Local edge computation, } \\
\text { 3D gradient calculation }\end{array}$ & Robust to noise and blurriness & $\begin{array}{l}\text { High frequency components } \\
\text { are not considered }\end{array}$ \\
\hline Zhou et al. (2014) & FREE surfer tool, SVM & $\begin{array}{l}\text { Combination of MMSE } \\
\text { and MRI improves classification }\end{array}$ & Five misclassification \\
\hline Rueda et al. (2014) & $\begin{array}{l}\text { Sailency brain pattern } \\
\text { extraction algorithm }\end{array}$ & Improved equal error rate & $\begin{array}{l}\text { It is secondary diagnostic } \\
\text { opinion }\end{array}$ \\
\hline Li Feng et al. ( 2016) & $\begin{array}{l}\text { Deep learning with drop } \\
\text { out for classification }\end{array}$ & $\begin{array}{l}\text { Improves the classification } \\
\text { for the small dataset }\end{array}$ & $\begin{array}{l}\text { Future focus is } \\
\text { on multikernel SVM }\end{array}$ \\
\hline
\end{tabular}


The classification algorithms developed for structural MR images involves generating Structural Abnormality Index (STAND) score based on a linear SVM (Vemuri et al., 2008). This technique adopts voxel selection method, by simultaneously examining all voxels related to pathology and feature reduction is performed by preserving the tissues which showed the variation. These tissue densities generate a STAND score which is a measure of normality and abnormality of a given structure. Including both demographic and genotypebased risk factors along with STAND score improves overall accuracy for classification.

Based on local frequency of an image, noise robust and rotation invariant texture features are extracted. The discrete fourier transform preserve some textural information in comparison to local binary function. A local frequency descriptor does not have an exponential growth of the patterns and it is more suitable for the images with the noise. The results show second frequency channel provides more information than the other higher frequency channels in curet and outex dataset for textural classification. In KTH-TIPS dataset, the fifth frequency channel gives more information for textural classification (Maani et al., 2013).

The joint statistical representation constituting the space and frequency domain is proposed for textural classification. The multi-resolution spatial filters are adopted for generating multi-scale spatial maps and local fourier transform is applied to obtain spectral maps. The spatial and spectral maps are quantized into different levels by global thresholding. These maps are jointly used to obtain space-frequency cooccurrence histogram (Song et al., 2014).

An automated unsupervised classification approach is used to distinguish AD subjects from NC. All the MRI images are subjected to pairwise registration by symmetric log-domain diffeomorphic demons algorithm (Long and Wyatt, 2010; Vercauteren et al., 2008). After the registration process, either the whole brain or the gray matter or white matter are considered for measuring the diffeomorphism. An affinity matrix is derived by the calculation of the deformation based riemannian distance between each pair of images. Then the spectral embedding of all the images is obtained by the calculated affinity values. Finally, the images are partitioned into sub-groups based on quick shift method.

Classification of morphological patterns using adaptive regional elements (COMPARE) (Fan et al., 2007) involves the development of tissue density maps, by subjecting it to warping technique. Then the volume of different tissues are measured to obtain the regions correlating to clinical variables. Then these regions are extracted by subjecting it to watershed segmentation and discriminative features are selected through SVM classifier. COMPARE attains high classification accuracy. An integrated framework is required to simultaneously extract and select effective regional features required for classification.

The multi-view learning technique (Zhu et al., 2015) extracts ROI and HOG (Histogram of Gradient) features from MRI images of brain. The extracted HOG feature is mapped to the ROI feature space by using two techniques: (i)Single-direction Mapping Multi-view Learning (SMML) utilizes a linear transformation matrix to transfer HOG features to the ROI feature space. (ii) In case of Directly Concatenating Multi-view Learning (DCML), the transfer is through an identity matrix. Both these features are subjected to SVM classifier for accurate identification of AD. Multiview features show better performance than single view features for classification of AD.

High-dimensional pattern classification approach (Davatzikos et al., 2009) is developed by combining all the brain regions together and identify regional volumes that develop a discriminative pattern in AD with the highest SPARE-AD score. Then the correlation between SPARE-AD and clinical variable are examined to aid the diagnosis.

The technique focuses on the automated method of Image analysis on OASIS-MIRIAD dataset (Rueda et al., 2014), by extracting saliency pattern of the brain due to neurodegenerative disease. The pattern developed is similar to the visual analysis made by radiologists. This begins with the generation of feature map, by integrating the following features like intensity, contrast, orientation and edges for different scales. For each combination of feature and scale, fully connected graph are constructed based on the dissimilarity and closeness. Then Markov chain is applied on the graph. It is followed by Bottom-up saliency fusion of feature-scale saliency maps. With the linear combination of feature-scale kernel and learned weights, master saliency maps are developed. Finally, discriminative relevance maps are generated by the fusion of all the master saliency maps. The regions showing the discriminative pattern in $\mathrm{AD}$ are cingulate gyrus, Entorhinal Cortex, and subcortical structures such as Amygdala, Hippocampus, Putamen which are same as it is obtained by the clinical studies. The metrics considered are accuracy, sensitivity, specificity, balanced accuracy, F measure, equal error rate measure. The proposed method results in improved classification. 


\section{Tools}

Tools are associated with specific functionalites, that are utilised to aid the process of Alzheimer's Disease detection. A brief overview of these tools are discussed in further paragraphs and functionalities of each tool are shown in Table 5.

FSL

Brain Extraction Tool (BET) (Smith, 2000) removes the non-brain tissues to increase the robustness of registration and to improve the performance of tissue segmentation. The tissue segmentation, bias field correction and partial volume effect at each voxel is performed using FAST (FMRIB's Automated Segmentation Tool). This is followed by image registration using FLIRT (FMRIB's Linear Image Registration Tool), which is based on intermodal cost function such as mutual information and correlation ratio that allows the robust registration of images from different modalities. After registration, the image is subjected to Structural Image Evaluation, using Normalization of Atrophy (SIENA). SIENA is an important tool used for clinical analysis, that involves quantitative measurement of change in shape and size (Smith et al., 2004).

\section{Free Surfer}

Zhou et al. (2014) proposed the free surfer technique by considering the volumetric variables and morphometric statistics for the classification of Alzheimer's Disease and Mild Cognitive Impairment. The ranking of variables is performed from low to high based on p-value obtained from student's t-test followed by the optimal selection of variables. in all experiments. In every iteration, the same The process is continued for 50 times by randomly assigning the variables and obtaining the average variables which are ranked in top three positions played a vital role in the classification. The volumetric variables with mini- mental state examination scores improve the accuracy in predicting the state of the disease. With respect to Alzheimer's Disease, both the hemisphere of the brain are atrophied. For Amnestic Mild Cognitive Impairment (aMCI), atrophy is developed on the right hemisphere of the brain and atrophy is developed on the left side for (naMCI) Non-Amnestic mild cognitive Impairment. The low ranked variable is not significant for discriminating the state of the disease.

\section{$3 D$ Slicer}

3D Slicer (Fedorov et al., 2012) is used as a platform for research and development. It provides automated segmentation and registration of images in various application domains and also has versatile visualization similar to radiology workstation. It is a comprehensive application that can be used for various tasks related to both the qualitative and quantitative inquiry of the multimodal medical imaging data. It provides an extensible architecture for customization and development of plug-ins that are operable across various platforms. The supported platforms are: Windows, Mac OS, Red Hat Linux and IRIX platforms.

\section{Neuro Quant Software Package}

The quality checking step is performed to find MR imaging sequence conforms to the specification required for an automated segmentation and it is followed by the correction of gradient nonlinearity and B1 field inhomogeneity. In the next step an active contour model is used for skull stripping. After the skull stripping, nonlinear registration aligns volumes to a probabilistic atlas for labeling an anatomy in the elderly subject. Intraclass Correlation Coefficient (ICC) and Pearson Product-Moment Correlation Coefficient are computed to evaluate the accuracy of segmentation (Brewer et al., 2009).

Table 5. Tools to aid Alzheimer's disease detection

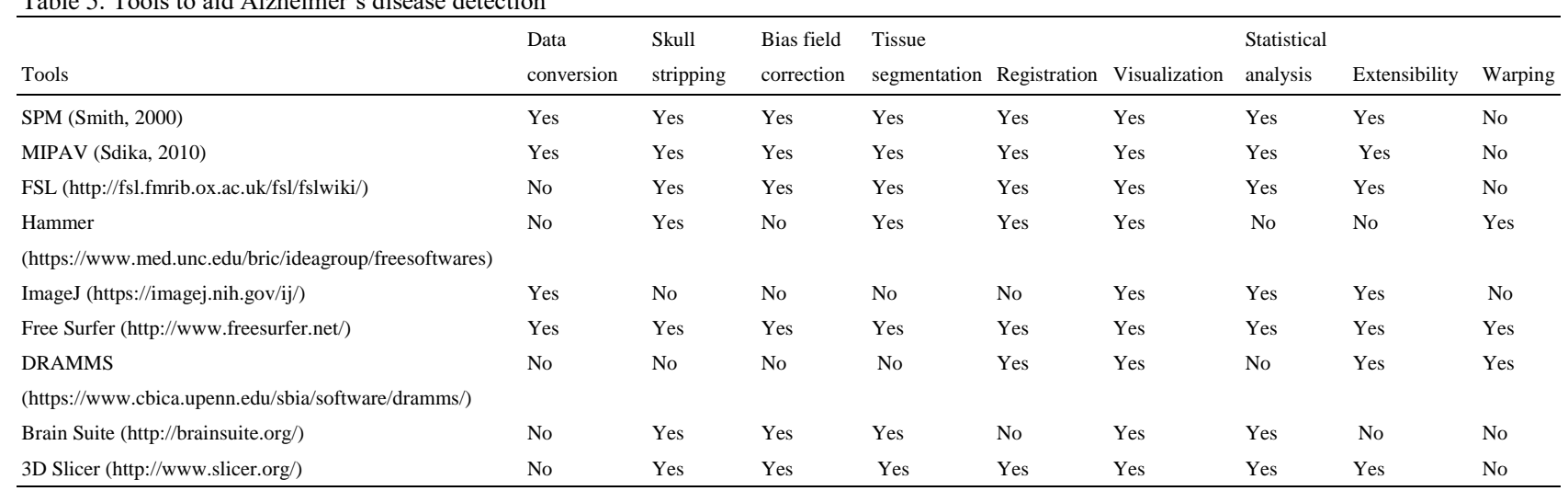




\section{Conclusion}

To identify sensitive biomarker and developing the advanced techniques are the key steps required for accurate detection of AD. Identification of relationship between neuropsychological tests and features from structural and functional images are necessary for accurate classification of different stages of AD. It is necessary to develop prognostic model that leads to accurate prediction of $\mathrm{MCI}$ to $\mathrm{AD}$ or $\mathrm{NC}$ to $\mathrm{AD}$ and it should be sensitive to heterogeneous dataset of $\mathrm{MCI}$ patients. Study on discover of multimodal biomarkers is still in progress for accurate diagnosis of AD. Numerous studies exclude the subjects, having incomplete longitudinal scores, thus the development of algorithms are necessary to complete the missing scores at different time points that aids to predict the future score required to investigate the present status of a patient.

\section{Author's Contributions}

All authors were involved in the manuscript preparation.

\section{Ethics}

This article is original and contains unpublished material. The corresponding author confirms that all of the other authors have read and approved the manuscript and there are no ethical issues involved.

\section{References}

Akadi, A.El., A. Amine, A. El Ouardighi and D. Aboutajdine, 2009. A new gene selection approach based on Minimum Redundancy-Maximum Relevance (MRMR) and Genetic Algorithm (GA). Proceedings IEEE/ACS International Conference Computer Systems Applications 69-75, 2009. 10-13 May 2009. DOI: 10.1109/AICCSA.2009.5069306

Aljabar, P., R.A. Heckemann, A. Hammers, J.V. Hajanl and D. Rueckert, 2009. Multi-atlas based segmentation of brain images: Atlas selection and its effect on accuracy. Neuroimage, 46: 726-738. DOI: 10.1016/j.neuroimage.2009.02.018

Anderson, V.M., J.M. Schott, J.W. Bartlett, K.K. Leung and D.H. Miller et al., 2012. Gray matter atrophy rate as a marker of disease progression in AD. Neurobiology Aging, 33: 1194-1202.

DOI: 10.1016/j.neurobiolaging.2010.11.001

Ashburner, J. and K.J. Friston, 2000. Voxel-based morphometry-the methods. Neuroimage, 11: 805-821. DOI: $10.1006 /$ nimg.2000.058

Ashburner, J., C. Hutton, R. Frackowiak, I. Johnsrude and C. Price et al., 1998. Identifying global anatomical differences: Deformation-based morphometry. Human Brain Mapping, 6: 348- 357.
Avants, B.B., C.L. Epstein, M. Grossman and J.C. Gee, 2008. Symmetric diffeomorphic image registration with cross-correlation: Evaluating automated labeling of elderly and neurodegenerative brain. Med. Image Analysis, 12: 26-41. DOI: 10.1016/j.media.2007.06.004

Bobinski, M., M.J. de Leon, A. Convit, S.D. Santi and J. Wegiel et al., 1999. MRI of entorhinal cortex in mild Alzheimer's disease. Lancet, 353: 38-40. DOI: $10.1016 / \mathrm{S} 0140-6736(05) 74869-8$

Brambilla, P., J.P. Hatch and J.C. Soares, 2008. Limbic changes identified by imaging in bipolar patients. Curr. Psych. Rep., 10: 505-509. PMID: 18980734

Brewer, J., S. Magda, C. Airriess and M. Smith, 2009. Fully-automated quantification of regional brain volumes for improved detection of focal atrophy in alzheimer disease. Am. J. Neuroradiol., 30: 578-580. DOI: 10.3174/ajnr.A1402

Bron, E.E., M. Smits, W.J. Niessen and S. Klein, 2015. Feature selection based on the SVM weight vector for classification of dementia. IEEE J. Biomed. Health Informatics, 19: 1617-1626. DOI: $10.1109 /$ JBHI.2015.2432832

Bro-Nielsen, M. and C. Gramkow, 1996. Fast fluid registration of medical images. Proceedings of the 4th International Conference Visualization in Biomedical Computing (VBC '96), Springer-Verlag, Denmark, pp: 267-276.

Cárdenas-Peña, D., D. Collazos-Huertas and G. Castellanos-Dominguez, 2016. Centered kernel alignment enhancing neural network pretraining for MRI-based dementia diagnosis. Comput. Math. Methods Med., 2016: 1-11.

DOI: $10.1155 / 2016 / 9523849$

Carmichael, O.T., H.A. Aizenstein, S.W. Davis, J.T. Becker and P.M. Thompson et al., 2005. Atlas-based hippocampus segmentation in Alzheimer's disease and mild cognitive impairment. Neuroimage, 27: 979-990. DOI: 10.1016/j.neuroimage.2005.05.005

Chan, T.F. and L.A. Vese, 2001. Active contours without edges. IEEE Trans. Image Proc., 10: 266-277. DOI: $10.1109 / 83.902291$

Chaplot, S., L. Patnaik and N. Jagannathan, 2006. Classification of magnetic resonance brain images using wavelets as input to support vector machine and neural network. Biomed. Signal Process. Control, 1: 86-92. DOI: 10.1016/j.bspc.2006.05.002

Chen, R. and E.H. Herskovits, 2010. Machine-learning techniques for building a diagnostic model for very mild dementia. Neuroimage, 52: 234-244. DOI: $10.1016 /$ j.neuroimage.2010.03.084

Cheng, B., M. Liu, D. Zhang, B.C. Munsell and D. Shen, 2015. Domain transfer learning for MCI Conversion prediction. IEEE Trans. Biomed. Eng., 62: 1805-1817. DOI: 10.1109/TBME.2015.2404809 
Chuang, K.S., H.L. Tzeng, S. Chen, J. Wu and T.J. Chen, 2006. Fuzzy c-means clustering with spatial information for image segmentation. Comput. Med. Imaging Graphics, 30: 19-15. DOI: 10.1016/j.compmedimag.2005.10.001

Chung, M., K. Worsley, T. Paus, C. Cherif and D. Collins et al., 2001. A unified statistical approach to deformation-based morphometry. NeuroImage, 14: 595-606. DOI: 10.1006/nimg.2001.0862

Cocosco, C.A., V. Kollokian, R.K.S. Kwan, G.B. Pike and A.C. Evans, 1997. Brainweb: Online interface to a 3D MRI simulated brain database. NeuroImage.

Costafreda, S.G., I.D. Dinov, Z. Tu, Y. Shi and C.Y. Liu et al., 2011. Automated hippocampal shape analysis predicts the onset of dementia in mild cognitive impairment. Neuroimage, 56: 212-219. DOI: 10.1016/j.neuroimage.2011.01.050

Coupé, P., J.V. Manjón, V. Fonov, J. Pruessner and M. Robles et al., 2011. Patch-based segmentation using expert priors: Application to hippocampus and ventricle segmentation. NeuroImage, 54: 940-954. DOI: 10.1016/j.neuroimage.2010.09.018

Crum, W.R., T. Hartkens and D. Hill, 2014. Non-rigid image registration: Theory and practice. Brit. J. Radiol., 77: S107-S208. DOI: 10.1259/bjr/25329214

Cui, Y., B. Liu, S. Luo, X. Zhen and M. Fan et al., 2011. Identification of conversion from mild cognitive impairment to Alzheimer's disease using multivariate predictors. Plos One, 6: e218-e296. DOI: 10.1371/journal.pone.0021896

Davatzikos, C., 1998. Mapping image data to stereotaxic spaces: Applications to brain mapping. Human Brain Mapping, 6: 334-338.

DOI:

$10.1002 /(\mathrm{SICI}) 1097-$ 0193(1998)6:5/6<334::AID-HBM2>3.0.CO;2-7

Davatzikos, C., A. Genc, D. Xu and S.M. Resnick, 2001.Voxel-based morphometry using the RAVENS maps: Methods and validation using simulated longitudinal atrophy. NeuroImage, 14: 1361-1369. DOI: 10.1006/nimg.2001.093

Davatzikos, C., F. Xu, Y. An, Y. Fan and S.M. Resnick, 2009. Longitudinal progression of Alzheimer's-like patterns of atrophy in normal older adults: The SPARE-AD index. Brain, 132: 2026-2035.

DOI: 10.1093/brain/awp09110.1093/brain/awp091

Davatzikos, C., P. Bhatt, L.M. Shaw, K.N. Batmanghelich and J.Q. Trojanowski, 2011. Prediction of MCI to AD conversion, via MRI, CSF biomarkers and pattern classification. Neurobiol. Aging, 32: 2322.e19-2322.e27. DOI: 10.1016/j.neurobiolaging.2010.05.023

De Jong, L.W., K. van der Hiele, I.M. Veer, J.J. Houwing and R.G.J. Westendorp et al., 2008. Strongly reduced volumes of putamen and thalamus in Alzheimer's disease: An MRI study. Brain, 131: 3277-3285. DOI: 10.1093/brain/awn278
De Leon, M.J., J. Golomb, A.E. George, A. Convit and C.Y. Tarshish et al., 1993. The radiologic prediction of Alzheimer disease: The atrophic hippocampal formation. Am. J. Neuroradiol., 14: 897-906. PMID: 8352162

Diaz, G., E. Romero, J.A. Hernández -Tamames, V. Molina and N. Malpica, 2010. Automatic classification of structural MRI for diagnosis of neurodegenerative diseases. Acta Biol. Colomb., 15: 165-180.

Duchesne, S., A. Caroli, C. Geroldi, C. Barillot and G.B. Frisoni et al., 2008. MRI-based automated computer classification of probable AD versus normal controls. IEEE Trans. Med. Imaging, 27: 509-520. DOI: 10.1109/TMI.2007.908685

Fan, Y., D. Shen and C. Davatzikos, 2005. Classification of structural images via high-dimensional image warping, robust feature extraction, and SVM. Proceedings of the International Conference on Medical Image Computing and Computer-Assisted Intervention, Sep. 10-14, Springer International Publishing, Quebec City, pp: 1-8.

Fan, Y., D. Shen, R.C. Gur, R.E. Gur and C. Davatzikos, 2007. Compare: Classification of morphological patterns using adaptive regional elements. IEEE Trans. Med. Imaging, 26: 193-105. DOI: 10.1109/TMI.2006.886812

Fan, Y., H. Rao, H. Hurt, J. Giannetta and M. Korczykowski et al., 2007. Multivariate examination of brain abnormality using both structural and functional MRI. NeuroImage, 36: 1189-1199.

DOI: 10.1016/j.neuroimage.2007.04.009

Fedorov, A., R. Beichel, J. Kalpathy-Cramer, J. Finet and J.C. Fillion- Robin et al., 2012. 3D slicer as an image computing platform for the quantitative imaging network. Magnetic Resonance Imaging, 30: 1323-1341. DOI: 10.1016/j.mri.2012.05.001

Filipovych, R., C. Davatzikos and N. Initiative, 2011. Semi-supervised pattern classification of medical images: Application to Mild Cognitive Impairment (MCI). NeuroImage, 55: 1109-1119. DOI: 10.1016/j.neuroimage.2010.12.066

Fox, N.C. and P.A. Freeborough, 1997. Brain atrophy progression measured from registered serial MRI: Validation and application to Alzheimer's disease. J. Magnetic Res. Imaging, 7: 1069-1075. DOI: $10.1002 /$ jmri.1880070620

Fox, N.C., S. Cousens, R. Scahill, R.J. Harvey and M.N. Rossor, 2000. Using serial registered brain magnetic resonance imaging to measure disease progression in alzheimer disease: Power calculations and estimates of sample size to detect treatment effects. Arch. Neurol., 57: 339-344. DOI: 10.1001/archneur.57.3.339 
Freeborough, P.A. and N.C. Fox, 1998. MR image texture analysis applied to the diagnosis and tracking of Alzheimer's disease. IEEE Trans. Med. Imaging, 17: 475-478. DOI: 10.1109/42.712137

Friston, K., J. Ashburner, C.D. Frith, J.B. Poline and J.D. Heather et al., 1995. Spatial registration and normalization of images. Human Brain Mapping, 3: 165-189. DOI: 10.1002/hbm.460030303

Gee, J.C., M. Reivich and R. Bajcsy, 1993. Elastically deforming $3 \mathrm{D}$ atlas to match anatomical brain images. J. Comp. Assist. Tomogr., 17: 225-236.

Gerig, G., M. Styner, M.E. Shenton and J.A. Lieberman, 2001. Shape versus size: Improved understanding of the morphology of brain structures. Med. Image Comput. ComputerAssisted Intervention, pp. 24-32.

Golland, P., W.E.L. Grimson and R. Kikinis, 1999. Statistical shape analysis using fixed topology skeletons: Corpus callosum study. Proceedings of the Biennial International Conference on Information Processing in Medical Imaging, Jun. 25-30, Boone, NC, USA, Springer International Publishing AG, pp: 382-387.

Golland, P., W.E.L. Grimson, M.E. Shenton and R. Kikinis, 2001. Deformation analysis for shape based classification. Proceedings of the International Conference on Information Processing in Medical Imaging, Jun. 25-30, Springer International Publishing AG, Boone, NC, USA, pp: 517-530.

Greenspan, H., A. Ruf and J. Goldberger, 2006. Constrained Gaussian mixture model framework for automatic segmentation of MR brain images. IEEE Trans. Med. Imaging, 25: 1233-1245. DOI: $10.1109 /$ TMI.2006.880668

Hackmack, K., F. Paul, M. Weygandt, C. Allefeld and J.D. Haynes et al., 2012. Multi-scale classification of disease using structural MRI and wavelet transform. Neuroimage, 62: 48-58.

DOI: 10.1016/j.neuroimage.2012.05.022

Hammouche, K., M. Diaf and P. Siarry, 2008. A multilevel automatic thresholding method based on a genetic algorithm for a fast image segmentation. Comput. Vision Image Understanding, 109: 163-175.

DOI: 10.1016/j.cviu.2007.09.001

Hartmann, S.L., M.H. Parks, P.R. Martin and B.M. Dawant, 1999. Automatic 3-d segmentation of internal structures of the head in MR images using a combination of similarity and free-form transformations. ii. validation on severely atrophied brains. IEEE Trans. Med. Imaging, 18: 917-926. DOI: $10.1109 / 42.811273$

Heckemann, R.A., S. Keihaninejad, P. Aljabar, K.R. Gray and C. Nielsen et al., 2011. Automatic morphometry in Alzheimer's disease and mild cognitive impairment. Neuroimage, 56: 2024-2037. DOI: 10.1016/j.neuroimage.2011.03.014
Hill, D.L., P.G. Batchelor, M. Holden and D.J. Hawkes, 2001. Medical image registration. Physics Med. Biol.

Holland, D., J.B. Brewer, D.J. Hagler, C. FennemaNotestine and A.M. Dale et al., 2009. Subregional neuroanatomical change as a biomarker for Alzheimer's disease. Proc. National Acad. Sci., 106: 20954-20959. DOI: 10.1073/pnas.0906053106

http://brainsuite.org/

http://fsl.fmrib.ox.ac.uk/fsl/fslwiki/

http://www.freesurfer.net/

http://www.slicer.org/

https://imagej.nih.gov/ij/

https://www.cbica.upenn.edu/sbia/software/dramms/

https://www.med.unc.edu/bric/ideagroup/freesoftwares/

$\mathrm{Hu}, \mathrm{K}$., Y. Wang, K. Chen, L. Hou and X. Zhang, 2016. Multi-scale features extraction from baseline structure MRI for MCI patient classification and AD early diagnosis. Neurocomputing, 175: 132-145. DOI: 10.1016/j.neucom.2015.10.043

Hu, S., P. Coupe, J.C. Pruessner and D.L. Collins, 2011. Appearance-based modeling for segmentation of hippocampus and amygdala using multi-contrast MR imaging. NeuroImage, 58: 549-559.

DOI: 10.1016/j.neuroimage.2011.06.054

Jack, C.R., M.A. Bernstein, N.C. Fox, P. Thompson and G. Alexander et al., 2008. The Alzheimer's Disease Neuroimaging Initiative (ADNI): MRI methods. J. Mag. Resonance Imaging, 27: 685-691. DOI: 10.1002/jmri.21049

Jain, A.K., J. Mao and K. Mohiuddin, 1996. Artificial neural networks: A tutorial. IEEE Comp., 29: 31-44. DOI: $10.1109 / 2.485891$

Joshi, S., P.D. Shenoy, K.R. Venugopal and L.M. Patnaik, 2010. Classification of neurodegenerative disorders based on major risk factors employing machine learning techniques. Int. J. Eng. Techn., 2: 350-356.

Joshi, S., P.D. Shenoy, P.L. Rrashmi, K.R. Venugopal and L. M. Patnaik, 2010. Classification of Alzheimer's disease and Parkinson's disease by using machine learning and neural network methods. Proceedings of the 2nd International Conference on Machine Learning and Computing, Feb. 9-11, IEEE Xplore Press, Bangalore, pp. 218-222.

DOI: $10.1109 /$ ICMLC.2010.45

Joshi, S., V. Simha, P.D. Shenoy, K.R. Venugopal and L.M. Patnaik, 2010. Classification and treatment of different stages of Alzheimer's disease using various machine learning methods. Int. J. Bioinformatics Res., 2: 44-52. DOI: 10.9735/0975-3087

Killiany, R.J., T. Gomez-Isla, M. Moss, R. Kikinis and T. Sandor et al., 2000. Use of structural magnetic resonance imaging to predict who will get Alzheimer's disease. Ann. Neurol., 47: 430-439. DOI: $10.1002 / 1531-8249(200004) 47: 4<430:$ AIDANA5>3.0.CO;2-I 
Kim, J., M.D.C. Valdes-Hernandez, N.A. Royle and J. Park, 2015. Hippocampal shape modeling based on a progressive template surface deformation and its verification. IEEE Trans. Med. Imaging, 34: 1242-1261. DOI: 10.1109/TMI.2014.2382581

Kumar, H.S., K. Raja, K.R. Venugopal and L.M. Patnaik, 2009. Automatic image segmentation using wavelets. Int. J. Comp. Sci. Network Security, 9: 305-313.

Kurani, A.S., D.H. Xu, J. Furst and D.S. Raicu, 2004. Cooccurrence matrices for volumetric data. Proceedings of the 7th International Conference Computer Graphics Imaging, Kauai, USA, pp. 447-452.

Lao, Z., D. Shen, Z. Xue, B. Karacali and S.M. Resnick et al., 2004. Morphological classification of brains via high-dimensional shape transformations and machine learning methods. Neuroimage, 21: 46-57.

DOI: 10.1016/j.neuroimage.2003.09.027

Li, F., L. Tran, K.H. Thung, S. Ji and D. Shen et al., 2015. A robust deep model for improved classification of AD/MCI patients. IEEE J. Biomed. Health Informatics, 19: 1610-1616. DOI: 10.1109/JBHI.2015.2429556

Li, M. and B. Yuan, 2005. 2D-LDA: A statistical linear discriminant analysis for image matrix. Pattern Recognition Lett., 26: 527-532. DOI: $10.1016 /$ j.patrec.2004.09.007

Li, X., L. Wang and E. Sung, 2005. A study of adaboost with SVM based weak learners. Proceddings of the IEEE International Joint Conference on Neural Networks, Jul. 31-Aug. 4, IEEE Xplore Press, Montreal, pp: 196-201. DOI: 10.1109/IJCNN.2005.1555829

Lin, X., F. Yang, L. Zhou, P. Yin and H. Kong et al., 2012. A support vector machine-recursive feature elimination feature selection method based on artificial contrast variables and mutual information. J. Chromatography, 910: 149-155. DOI: 10.1016/j.jchromb.2012.05.020

Liu, F., L. Zhou, C. Shen and J. Yin, 2014. Multiple kernel learning in the primal for multimodal Alzheimer's disease classification. IEEE J. Biomed. Health Informatics, 18: 984-990. DOI: $10.1109 /$ JBHI.2013.2285378

Liu, M., D. Zhang, E. Adeli and D. Shen, 2015. Inherent Structure-based multiview learning with multitemplate feature representation for Alzheimer's disease diagnosis. 1EEE Trans. Biomed. Eng., 63: 1473-1482. DOI: 10.1109/TBME.2015.2496233

Liu, S., S. Liu, W. Cai, H. Che and S. Pujol et al., 2015. Multimodal neuroimaging feature learning for multiclass diagnosis of alzheimer's disease. IEEE Trans. Biomed. Eng., 62: 1132-1140. DOI: 10.1109/TBME.2014.2372011
Liu, Y., L. Teverovskiy, O. Carmichael, R. Kikinis and M. Shenton et al., 2004. Discriminative MR image feature analysis for automatic schizophrenia and Alzheimer's Disease classification. Proceedings of the International Conference on Medical Image Computing and Computer-Assisted Intervention, Sep. 10-14, Springer International Publishing AG, pp: 393-401.

Liu, Y., L. Teverovskiy, O.L. Lopez, H. Aizenstein and C. Meltzer et al., 2007. Dicovery of "Biomarkers" for Alzheimer's Disease prediction from structural MR images. Proceedings of the 4th International Symposium on Biomedical Imaging, Apr. 12-15, IEEE Xplore Press, Arlington, pp: 1344-1347.

DOI: $10.1109 /$ ISBI.2007.357109

Long, X. and C. Wyatt, 2010. An automatic unsupervised classification of MR images in Alzheimer's disease. Proceedings of the IEEE Conference on Computer Vision and Pattern Recognition, Jun. 13-18, IEEE Xplore Press, San Francisco, CA, USA, pp: 2910-2917. DOI: $10.1109 /$ CVPR.2010.5540031

Lötjönen, J.M., R. Wolz, J.R. Koikkalainen, L. Thurfjell and G. Waldemar et al., 2010. Fast and robust multiatlas segmentation of brain magnetic resonance images. Neuroimage, 49: 2352-2365. DOI: 10.1016/j.neuroimage.2009.10.026

Maani, R., S. Kalra and Y.H. Yang, 2013. Noise robust rotation invariant features for texture classification. Pattern Recognition, 46: 2103-2116. DOI: $10.1016 /$ j.patcog.2013.01.014

Maani, R., S. Kalra and Y.H. Yang, 2014. Robust volumetric texture classification of magnetic resonance images of the brain using local frequency descriptor. IEEE Trans. Image Proces., 23: 4625-4636. DOI: $10.1109 /$ TIP.2014.2351620

Maes, F., A. Collignon, D. Vandermeulen, G. Marcha and P. Suetens, 1997. Multimodality image registration by maximization of mutual information. IEEE Trans. Med. Imaging, 16: 187-198. DOI: $10.1109 / 42.563664$

Malone, I.B., D. Cash, G.R. Ridgway, D.G. MacManus and S. Ourselin et al., 2013. MIRIAD-Public release of a multiple time point Alzheimer's MR imaging dataset. NeuroImage, 70: 33-36. DOI: 10.1016/j.neuroimage.2012.12.044

Marcus, D.S.,T.H. Wang, J. Parker, J.G. Csernansky and J.C. Morris et al., 2007. Open Access Series of Imaging Studies (OASIS): Cross-sectional MRI data in young, middle aged, nondemented and demented older adults. J. Cognitive Neurosci., 19: 1498-1507. DOI: 10.1162/jocn.2007.19.9.1498 
Mayer, A. and H. Greenspan, 2009. An adaptive meanshift framework for MRI brain segmentation. IEEE Trans. Med. Imaging, 28: 1238-1250. DOI: 10.1109/TMI.2009.2013850

Mazziotta, J., A. Toga, A. Evans, P. Fox and J. Lancaster et al., 2001. A probabilistic atlas and reference system for the human brain: International Consortium for Brain Mapping (ICBM). Philosophical Trans. Royal Society London B: Biol. Sci., 356: 1293-1322. DOI: 10.1098/rstb.2001.0915

Mechelli, A., C.J. Price, K.J. Friston and J. Ashburner, 2005. Voxel-based morphometry of the human brain: Methods and applications. Curr. Med. Imaging Rev., 1: 105-113. DOI: $10.2174 / 1573405054038726$

Morra, J.H., Z. Tu, L.G. Apostolova and A.E. Green et al., 2008. Validation of a fully automated 3D hippocampal segmentation method using subjects with Alzheimer's disease, mild cognitive impairment and elderly controls. Neuroimage, 43: 59-68.

DOI: 10.1016/j.neuroimage.2008.07.003

Nachar, N., 2008. The mann-whitney u: A test for assessing whether two independent samples come from the same distribution. Tutorials Quantitative Methods Psychology, 4: 13-20. DOI: 10.20982/tqmp.04.1.p013

Nestor, P.J., P. Scheltens and J.R. Hodges, 2004. Advances in the early detection of Alzheimer's disease. Nature Med.

Nestor, S.M., R. Rupsingh, M. Borrie, M. Smith and V. Accomazzi et al., 2008. Ventricular enlargement as a possible measure of Alzheimer's disease progression validated using the Alzheimer's disease neuroimaging initiative database. Brain, 131: 2443-2454. DOI: 10.1093/brain/awn146

Niyogi, X., 2004. Locality preserving projections. The University of Chicago.

Pieperhoff, P., L. Hömke, F. Schneider, U. Habel and N.J. Shah et al., 2008. Deformation field morphometry reveals age-related structural differences between the brains of adults up to 51 years. J. Neurosci., 28: 828842. DOI: 10.1523/JNEUROSCI.3732-07.2008

Qiu, A., C. Fennema-Notestine, A.M. Dale, M.I. Miller and N. Initiative et al., 2009. Regional shape abnormalities in mild cognitive impairment and alzheimer's disease. Neuroimage, 45: 656-661. DOI: 10.1016/j.neuroimage.2009.01.013

Ramírez, J., J. Górriz, F. Segovia, R. Chaves and D. Salas-Gonzalez et al., 2010. Computer aided diagnosis system for the Alzheimer's disease based on partial least squares and random forest SPECT image classification. Neurosci. Let., 472: 99-103. DOI: 10.1016/j.neulet.2010.01.056

Rexilius, J., S.K. Warfield, C.R. Guttmann, X. Wei and R. Benson et al., 2001. A novel nonrigid registration algorithm and applications. Proceedings of the International Conference on Medical Image Computing and Computer-Assisted Intervention Oct. 05, Springer International Publishing AG, USA, pp: 923-931.
Ribbens, A., J. Hermans, F. Maes, D. Vandermeulen and P. Suetens, 2014. Unsupervised segmentation, clustering and groupwise registration of heterogeneous populations of brain MR images. IEEE Trans. Med. Imaging, 33: 201-224.

DOI: 10.1109/TMI.2013.2270114

Rohlfing, T., R. Brandt, R. Menzel and C.R. Maurer, 2004. Evaluation of atlas selection strategies for atlasbased image segmentation with application to confocal microscopy images of bee brains. NeuroImage, 21: 1428-1442. DOI: 10.1016/j.neuroimage.2003.11.010

Rueckert, D., L.I. Sonoda, C. Hayes, D.L. Hill and M.O. Leach et al., 1999. Nonrigid registration using free-form deformations: Application to breast MR images. IEEE Trans. Med. Imaging, 18: 712-721. DOI: 10.1109/42.796284

Rueda, A., F.A. González and E. Romero, 2014. Extracting salient brain patterns for imagingbased classification of neurodegenerative diseases. IEEE Trans. Med. Imaging, 33: 1262-1274.

DOI: 10.1109/TMI.2014.2308999

Sabuncu, M.R., B.T. Yeo, K. Van Leemput, B. Fischl and P. Golland, 2010. A Generative model for image segmentation based on label fusion. IEEE Trans. Med. Imaging, 29: 1714-1729.

DOI: 10.1109/TMI.2010.2050897

San, J., H.Y. Lee, H.J. Shin, Y.K. Kim and N.Y. Jang et al., 2016. Combined effects of physical exercise and education on age-related cortical thinning in cognitively normal individuals. Sci. Reports, 6: 242-284. DOI: 10.1038/srep24284

Sanroma, G., Wu, G., Gao, Y. and Shen, D., 2014. Learning to rank atlases for multiple-atlas segmentation. IEEE transactions on medical imaging, 33(10), pp.1939-1953.

Savio, A., M. García-Sebastián, M. Graña and J. Villanúa, 2009. Results of an adaboost approach on Alzheimer's disease detection on MRI. Bioinspired Applic. Artificial Natural Comput., pp. 114-123.

Scahill, R.I., J.M. Schott, J.M. Stevens, M.N. Rossor and N.C. Fox, 2002. Mapping the evolution of regional atrophy in Alzheimer's disease: Unbiased analysis of fluid-registered serial MRI. Proc. Nat. Acad. Sci., 99: 4703-4707. DOI: 10.1073/pnas.052587399

Schmitter, D., A. Roche, B. Maréchal, D. Ribes and A. Abdulkadir et al., 2015. An evaluation of volume-based morphometry for prediction of mild cognitive impairment and Alzheimer's disease. NeuroImage: Clinical, 7: 71-17.

DOI: $10.1016 /$ j.nicl.2014.11.001

Sdika, M., 2010. Combining atlas based segmentation and intensity classification with nearest neighbor transform and accuracy weighted vote. Med. Image Anal., 14: 219-226. DOI: 10.1016/j.media.2009.12.004 
Segovia, F., J. Górriz, J. Ramírez, D. Salas-Gonzalez and I. Álvarez et al., 2012. A comparative study of feature extraction methods for the diagnosis of Alzheimer's disease using the ADNI database. Neurocomputing, 75: 64-71.

DOI: $10.1016 /$ j.neucom.2011.03.050

Senjem, M.L., J.L. Gunter, M.M. Shiung, R.C. Petersen and C.R. Jack, 2005. Comparison of different methodological implementations of Voxel-based morphometry in neurodegenerative disease. Neuroimage, 26: 600-608.

DOI: 10.1016/j.neuroimage.2005.02.005

Senthilkumaran, N. and R. Rajesh, 2009. Edge detection techniques for image segmentation - a survey of soft computing approaches. Int. J. Recent Trends Eng. Technol., 1: 250-254. DOI: 01.IJRTET.01.02.1474

Shenoy, P.D., K.G. Srinivasa, K.R. Venugopal and L.M. Patnaik, 2005. Dynamic association rule mining using genetic algorithms. J. Intelligent Data Anal., 9: 439-453.

Shenton, M., R. Kikinis, R. McCarley, P. Saiviroonporn and H. Hokama et al., 1995. Harvard brain atlas: A teaching and visualization tool. Proceedings of the Biomedical Visualization, Oct. 30-Nov. 3, IEEE Xplore press, Atlanta USA.

DOI: 10.1109/BIOVIS.1995.528700

Smith, S.M., 2000. BET: Brain extraction tool. Oxford Centre for Functional Magnetic Resonance Imaging of the Brain, Department of Clinical Neurology Oxford University, John Radcliffe Hospital, Headington. UK,

Smith, S.M., M. Jenkinson, M.W. Woolrich, C.F. Beckmann and T.E. Behrens et al., 2004. Advances in functional and structural MR image analysis and implementation as FSL. Neuroimage, 23: S208- S219. DOI: 10.1016/j.neuroimage.2004.07.051

Song, T., H. Li, B. Zeng and M. Gabbouj, 2014. Texture classification using joint statistical representation in space-frequency domain with local quantized patterns. Proceedings of the IEEE International Symposium on Circuits and Systems, Jun. 1-15, IEEE Xplore Press, Melbourne VIC, Australia, pp: 886-889.

DOI: $10.1109 /$ ISCAS.2014.6865278

Srinivasa, K.G., A. Singh, A.O. Thomas, K.R. Venugopal and L.M. Patnaik, 2005. Generic feature extraction for classification using fuzzy c - means clustering. Proceedings of the 3rd International Conference on Intelligent Sensing and Information Processing, Dec. 14-17, IEEE Xplore Press, Bangalore, India, pp: 33-38.

DOI: 10.1109/ICISIP.2005.1619409
Stoeckel, J., N. Ayache, G. Malandain, P.M. Koulibaly and K.P. Ebmeier et al., 2004. Automatic classification of SPECT images of Alzheimer's disease patients and control subjects. Proceedings of the International Conference on Medical Image Computing and Computer-Assisted Intervention, Sep. 10-14, Springer International Publishing AG, Quebec City, QC, Canada, pp: 654-662.

Suk, H.I., S.W. Lee, D. Shen and N. Initiative et al., 2015. Latent feature representation with stacked auto-encoder for AD/MCI diagnosis. Brain Struct. Funct., 220: 841-859. DOI: $10.1007 / \mathrm{s} 00429-013-0687-3$

Tang, S., Y. Fan, G. Wu, M. Kim and D. Shen, 2009. RABBIT: Rapid alignment of brains by building intermediate templates. NeuroImage, 47: 12771287. DOI: 10.1016/j.neuroimage.2009.02.043

Tang, X., D. Holland, A.M. Dale, L. Younes and M.I. Miller, 2014. Shape abnormalities of subcortical and ventricular structures in mild cognitive impairment and Alzheimer's disease: Detecting, quantifying and predicting. Human Brain Mapping, 35: 3701-3725. DOI: $10.1002 / \mathrm{hbm} .22431$

Teipel, S.J., C. Born, M. Ewers, A.L. Bokde and M.F. Reiser et al., 2007. Multivariate deformationbased analysis of brain atrophy to predict Alzheimer's disease in mild cognitive impairment. Neuroimage, 38: 13-24. DOI: 10.1016/j.neuroimage.2007.07.008

Teipel, S.J., W. Bayer, G.E. Alexander, A.L.W. Bokde and Y. Zebuhr et al., 2003. Regional pattern of hippocampus and corpus callosum atrophy in Alzheimer's disease in relation to dementia severity: Evidence for early neocortical degeneration. Neurobiol. Aging, 24: 85-94. DOI: $10.1016 / \mathrm{S} 0197-4580(02) 00044-1$

Testa, C., M.P. Laakso, F. Sabattoli, R. Rossi and A. Beltramello et al., 2004. A Comparison between the accuracy of voxel-based morphometry and hippocampal volumetry in Alzheimer's disease. J. Magnetic Res. Imaging, 19: 274-282. DOI: $10.1002 /$ jmri.20001

Thompson, P.M., K.M. Hayashi, G.I. de Zubicaray, A.L. Janke and S.E. Rose et al., 2004. Mapping hippocampal and ventricular change in alzheimer disease. Neuroimage, 22: 1754-1766. DOI: $10.1016 /$ j.neuroimage.2004.03.040

Tobias, O.J. and R. Seara, 2002. Image segmentation by histogram thresholding using fuzzy sets. IEEE Trans. Image Proc., 11: 1457-1465. DOI: 10.1109/TIP.2002.806231

Tu, Z. and X. Bai, 2010. Auto-context and its application to high-level vision tasks and 3D brain image segmentation. IEEE Trans. Pattern Anal. Machine Intelligence, 32: 1744-1757. DOI: $10.1109 /$ TPAMI.2009.186 
Van Hulle, M.M., 2012. Self-Organizing Maps. In: Handbook Natural Computing, Rozenberg, G., T. Bäck and J.N. Kok (Eds.), Springer Berlin Heidelberg, Berlin [Germany], ISBN-10: 3540929118, pp: 585-622.

Van Leemput, K., F. Maes, D. Vandermeulen and P. Suetens, 2003. A unifying framework for partial volume segmentation of brain MR images. IEEE Trans. Med. Imaging, 22: 105-119. DOI: 10.1109/TMI.2002.806587

Vemuri, P., J.L. Gunter, M.L. Senjem, J.L. Whitwell and K. Kantarci et al., 2008. Alzheimer's disease diagnosis in individual subjects using structural MR images: Validation studies. Neuroimage, 39: 1186- 1197. DOI: 10.1016/j.neuroimage.2007.09.073

Venugopal, K.R., K.G. Srinivasa and L.M. Patnaik, 2009. Soft Computing for Data Mining Applications. 1st Edn., Springer Science \& Business Media, Berlin, ISBN-10: 3642001920, pp: 341.

Vercauteren, T., X. Pennec, A. Perchant and N. Ayache, 2008. Symmetric log-domain diffeomorphic registration: A demons-based approach. Proceedings of the International Conference on Medical Image Computing and Computer-Assisted Intervention, Sep. 10-14, Springer International Publishing AG, Quebec City, QC, Canada, pp: 754-761.

Vibha, L., G. Harshavardhan, K. Pranaw, P.D. Shenoy and K.R. Venugopal et al., 2006. Classification of mammograms using decision trees. Proceedings of the 10th International Database Engineering and Applications Symposium, Dec. 11-14, IEEE Xplore Press, Delhi, India, pp. 263-266.

DOI: 10.1109/IDEAS.2006.14

Vibha, L., G.M. Harshavardhan, K. Pranaw, P.D. Shenoy and K.R. Venugopal et al., 2007. Lesion detection using segmentation and classification of mammograms. Proceedings of the 25th IASTED International Multi-Conference: Artificial Intelligence and Applications, Feb. 12-14, ACTA Press Anaheim, CA, USA, Innsbruck, Austria, pp: 311-316.

Vincent, P., H. Larochelle, I. Lajoie, Y. Bengio and P.A. Manzagol, 2010. Stacked denoising autoencoders: learning useful representations in a deep network with a local denoising criterion. J. Machine Learn. Res., 11: 3371-3408.

Walhovd, K., A. Fjell, J. Brewer, L. McEvoy and C. Fennema-Notestine et al., 2010. Combining MR imaging, positron-emission tomography and CSF biomarkers in the diagnosis and prognosis of Alzheimer disease. Am. J. Neuroradiol., 31: 347-354. DOI: doi.org/10.3174/ajnr.A1809

Wang, H., S.R. Das, J.W. Suh, M. Altinay and J. Pluta et al., 2011. A learning-based wrapper method to correct systematic errors in automatic image segmentation: consistently improved performance in hippocampus, cortex and brain segmentation. NeuroImage, 55: 968-985.

DOI: 10.1016/j.neuroimage.2011.01.006
Wang, J., G. de Haan, D. Unay, O. Soldea and A. Ekin, 2009. Voxel-based discriminant map classification on brain ventricles for Alzheimer's disease. Proceedings of the Medical Imaging 2009: Image Processing, Feb. 7-12, SPIE Digital Library, pp. 72591W-72591W. DOI: $10.1117 / 12.810908$

Warfield, S.K., K.H. Zou and W.M. Wells, 2004. Simultaneous truth and performance level estimation (staple): An algorithm for the validation of image segmentation. IEEE Trans. Med. Imaging, 23: 903-921.

DOI: 10.1109/TMI.2004.828354

Webb, J., A. Guimond, P. Eldridge, D. Chadwick and J. Meunier et al., 1999. Automatic detection of hippocampal atrophy on magnetic resonance images. Magnetic Resonance Imaging, 17: 1149-1161. DOI: 10.1016/S0730-725X(99)00044-2

Wells, W.M., P. Viola, H. Atsumi, S. Nakajima and R. Kikinis, 1996. Multi-modal volume registration by maximization of mutual information. Med. Image Anal., 1: 35-51.

DOI: 10.1016/S1361-8415(01)80004-9

Whitwell, J.L., 2009. Voxel-based morphometry: An automated technique for assessing structural changes in the brain. J. Neurosci., 29: 9661-9664. DOI: 10.1523/JNEUROSCI.2160-09.2009

Whitwell, J.L., S.A. Przybelski, S.D. Weigand, D.S. Knopman and B.F. Boeve et al., 2007. 3D maps from multiple MRI illustrate changing atrophy patterns as subjects progress from mild cognitive impairment to Alzheimer's disease. Brain, 130: 1777-1786. DOI: 10.1093/brain/awm112

Wold, S., K. Esbensen and P. Geladi, 1987. Principal component analysis. Chemometrics Intelligent Laboratory Syst., 2: 37-52.

DOI: 10.1016/0169-7439(87)80084-9

Wu, M., C. Rosano, P. Lopez-Garcia, C.S. Carter and H.J. Aizenstein, 2007. Optimum template selection for atlas-based segmentation. Neuroimage, 34: 1612-1618. DOI: 10.1016/j.neuroimage.2006.07.050

Xue, Z., D. Shen and C. Davatzikos, 2004. Determining correspondence in 3-D MR brain images using attribute vectors as morphological signatures of voxels. IEEE Trans. Med. Imaging, 23: 1276-1291. DOI: 10.1109/TMI.2004.834616

Yang, S.T., J.D. Lee, T.C. Chang, C.H. Huang and J.J. Wang et al., 2013. Discrimination between Alzheimer's disease and mild cognitive impairment using SOM and PSO-SVM. Comput. Math. Methods Med., 2013: 1-11. DOI: 10.1155/2013/253670

Yazdani, S., R. Yusof, A. Karimian, Y. Mitsukira and A. Hematian, 2016. Automatic region-based brain classification of MRI-T1 data. PloS One, 11: 1-19. DOI: $10.1371 /$ journal.pone.0151326 
Zarpalas, D., P. Gkontra, P. Daras and N. Maglaveras, 2014. Gradient-Based reliability maps for ACMbased segmentation of hippocampus. IEEE Trans. Biomed. Eng., 61: 1015-1026.

DOI: 10.1109/TBME.2013.2293023

Zeng, X., L.H. Staib, R.T. Schultz and J.S. Duncan, 1999. Segmentation and measurement of the cortex from 3-D MR images using coupledsurfaces propagation. IEEE Trans. Med. Imaging, 18: 927-937. DOI: 10.1109/42.811276

Zhang, D. and D. Shen, 2011. Semi-supervised multimodal classification of Alzheimer's disease. Proceedings of the IEEE International Symposium on Biomedical Imaging: From Nano to Macro, Mar. 30-Apr. 2, IEEE Xplore Press, Chicago, IL, USA, pp: 1628-1631. DOI: 10.1109/ISBI.2011.5872715

Zhang, D., D. Shen and N. Initiative et al., 2012. Multi-modal multi-task learning for joint prediction of multiple regression and classification variables in Alzheimer's disease. NeuroImage, 59: 895-907.

DOI: 10.1016/j.neuroimage.2011.09.069

Zhang, D., Y. Wang, L. Zhou, H. Yuan and D. Shen et al., 2011. Multimodal classification of Alzheimer's disease and mild cognitive impairment. Neuroimage, 55: 856-867.

DOI: $10.1016 /$ j.neuroimage.2011.01.008
Zhang, N., S. Ruan, S. Lebonvallet, Q. Liao and Y. Zhu, 2009. Multi-kernel SVM based classification for brain tumor segmentation of MRI multisequence. Proceedings of the 16th IEEE International Conference on Image Processing, Nov. 7-10, IEEE Xplore Press, Cairo, Egypt, pp: 3373-3376. DOI: 10.1109/ICIP.2009.5413878

Zhang, Y., Z. Dong, L. Wu and S. Wang, 2011. A hybrid method for MRI brain image classification. Expert Syst. Applic., 38: 1004910053. DOI: 10.1016/j.eswa.2011.02.012

Zhou, Q., M. Goryawala, M. Cabrerizo, J. Wang and W. Barker et al., 2014. An optimal decisional space for the classification of Alzheimer's disease and mild cognitive impairment. IEEE Trans. Biomed. Eng., 61: 2245-2253. DOI: 10.1109/TBME.2014.2310709

Zhu, X., H.I. Suk, S.W. Lee and D. Shen, 2016. Subspace regularized sparse multitask learning for multiclass neurodegenerative disease identification. IEEE Trans. Biomed. Eng., 63: 607-618. DOI: 10.1109/TBME.2015.2466616

Zhu, X., H.I. Suk, Y. Zhu, K.H. Thung and G. Wu et al., 2015. Multi-view classification for identification of Alzheimer's disease. Machine Learn. Med. Imaging. 